\title{
Global Well-Posedness for a Class of Kirchhoff-Type Wave System
}

\author{
Xiaoli Jiang and Xiaofeng Wang \\ Department of Mathematics, Bohai University, Jinzhou, Liaoning 121013, China \\ Correspondence should be addressed to Xiaoli Jiang; jxls309@163.com
}

Received 6 June 2017; Revised 9 August 2017; Accepted 8 November 2017; Published 7 December 2017

Academic Editor: Rehana Naz

Copyright (c) 2017 Xiaoli Jiang and Xiaofeng Wang. This is an open access article distributed under the Creative Commons Attribution License, which permits unrestricted use, distribution, and reproduction in any medium, provided the original work is properly cited.

\begin{abstract}
In recent years, the small initial boundary value problem of the Kirchhoff-type wave system attracts many scholars' attention. However, the big initial boundary value problem is also a topic of theoretical significance. In this paper, we devote oneself to the well-posedness of the Kirchhoff-type wave system under the big initial boundary conditions. Combining the potential well method with an improved convex method, we establish a criterion for the well-posedness of the system with nonlinear source and dissipative and viscoelastic terms. Based on the criteria, the energy of the system is divided into different levels. For the subcritical case, we prove that there exist the global solutions when the initial value belongs to the stable set, while the finite time blow-up occurs when the initial value belongs to the unstable set. For the supercritical case, we show that the corresponding solution blows up in a finite time if the initial value satisfies some given conditions.
\end{abstract}

\section{Introduction}

This paper studies the initial boundary value problem for the following Kirchhoff-type wave system:

$$
\begin{array}{r}
u_{t t}-M(t) \Delta u+\int_{0}^{t} g_{1}(t-s) \Delta u d s+g\left(u_{t}\right) \\
=f_{1}(u, v) \quad \text { in } \Omega \times(0,+\infty), \\
v_{t t}-M(t) \Delta v+\int_{0}^{t} g_{2}(t-s) \Delta u d s+g\left(v_{t}\right)=f_{2}(u, v) \\
\text { in } \Omega \times(0,+\infty),
\end{array}
$$

$u(x, 0)=u_{0}(x)$

$u_{t}(x, 0)=u_{1}(x)$

in $\Omega$,

$v(x, 0)=v_{0}(x)$,

$v_{t}(x, 0)=v_{1}(x)$

$$
u(x, t)=v(x, t)=0 \quad \text { on } \Gamma_{1} \times[0,+\infty),
$$

where $\Omega$ is a bounded domain in $\mathbb{R}^{n}(n=1,2,3)$ with a smooth boundary $\partial \Omega, g\left(u_{t}\right)=\left|u_{t}\right|^{p-1} u_{t}, g\left(v_{t}\right)=\left|v_{t}\right|^{q-1} v_{t}$, $M(r)$ is a nonnegative $C^{1}$ function like $M(t)=m_{0}+\alpha\left(\|\nabla u\|^{2}+\right.$ $\left.\|\nabla v\|^{2}\right)^{\gamma}$, with $m_{0} \geq 0, \alpha \geq 0, m_{0}+\alpha>0, \gamma>0$, and $g_{1}, g_{2}$ : $R^{+} \rightarrow R^{+}, f_{i}(\cdot, \cdot): R^{2} \rightarrow R, i=1,2$, are given functions which will be specified later.

1.1. Historical Research. Kirchhoff-type wave system with nonlinear source and dissipative and viscoelastic terms have various applications in the field of physics and mechanics, which is the model to describe the motion of deformable solids. A single Kirchhoff-type wave equation is proposed:

$$
u_{t t}-M\left(\|\nabla u\|^{2}\right) \Delta u+\int_{0}^{t} g(t-s) \Delta u d s+h\left(u_{t}\right)
$$

$$
=f(u) \text { in } \Omega \times(0,+\infty) \text {, }
$$

and (6) has its roots for the small amplitude vibrations of a string when $g=0$ and $n=1$, but the tension of the string can not be ignored (see, e.g., Carrier et al. [1]). While (6) is used to describe the dynamics of an elastic string with fading 
memory when $g \neq 0$, this equation shows that the dynamic equilibrium of the object depends on both the present state of deformation and the history of the deformation gradient. Pohozaev and Tesei [2] proved that the solution exists in time if the datum satisfy an analytic-type condition for the case $g=$ 0 . This result of the case $g \neq 0$ was extended by Torrejon and Yong [3]; they obtained the existence of weakly asymptotic stable solution. Later, Munoz Rivera [4] showed the existence of global solutions for small initial value and the exponential decay of the total energy. Then $\mathrm{Wu}$ and Tsai [5] established the global existence and energy decay under the assumption $g^{\prime}(t) \leq-r g(t), h\left(u_{t}\right)=-\Delta u_{t}$. Recently, this decay estimate was improved for a weaker condition on $g^{\prime}(t) \leq 0$ in [6].

Problem (6) is simplified to the following format without viscoelastic term:

$$
u_{t t}-M\left(\|\nabla u\|^{2}\right) \Delta u+h\left(u_{t}\right)=f(u)
$$

$$
\text { in } \Omega \times(0,+\infty) \text {, }
$$

and some results of (7) concerning global well-posedness have been established in [7-11] for the case of $M \equiv 1$. The above problem without source and dissipative terms is called Kirchhoff-type equation when $M$ is not a constant function, which was first introduced by Kirchhoff [12]; it describes the nonlinear vibrations of an elastic string. up to now, there are numerous results related to global well-posedness, including global existence, decay result, and blow-up properties; we refer the reader to [13-17].

Most recently Xu and Yang considered the initial boundary value problem of the following equation in [18]; they gave a blow-up result under supercritical energy:

$$
\begin{aligned}
u_{t t} & -\Delta u+\int_{0}^{t} g(t-\tau) \Delta u(\tau) d \tau-\Delta u_{t}-\Delta u_{t t}+u_{t} \\
& =|u|^{p-1} u .
\end{aligned}
$$

Wave system such as (1) and (2) goes back to Reed [19] who proposed a similar system, but it does not contain $M(t)=m_{0}+\alpha\left(\|\nabla u\|^{2}+\|\nabla v\|^{2}\right)^{\gamma}$ and the viscoelastic terms $\int_{0}^{t} g_{1}(t-s) \Delta u d s, \int_{0}^{t} g_{2}(t-s) \Delta u d s$. Subsequently, concerning blow-up and nonexistence, results in wave systems were discussed. Agre and Rammaha [20] studied the following concrete system:

$$
\begin{gathered}
u_{t t}-\Delta u+\left|u_{t}\right|^{m-1} u_{t}=f_{1}(u, v), \\
v_{t t}-\Delta v+\left|v_{t}\right|^{r-1} v_{t}=f_{2}(u, v),
\end{gathered}
$$

in $\Omega \subset \mathbb{R}^{n} \times(0, T)(n=1,2,3)$ with initial and boundary conditions of Dirichlet type, where $f_{1}(u, v)$ and $f_{2}(u, v)$ satisfy (A1) and (A2). They obtained several results concerning global well-posedness of a weak solution and showed that any weak solution blows up in finite time at negative initial energy. Afterwards, Alives et al. made further efforts as regards (9) in [21]. They obtained the global existence, uniform decay rates, and blow-up of solutions in finite time by involving the Nehari manifold when the initial energy is nonnegative and less than the mountain pass level value.
And this blow-up result was improved by Said-Houari [22] when the initial data are large enough. In [23], Rammaha and Sakuntasathien studied a more general case of (9) by degenerating damping terms. Several results on the existence of local and global solutions as well as uniqueness are obtained by considering the constraint on the parameters of the system. Furthermore, they proved that the weak solutions blow up in finite time whenever the initial energy is negative and the exponent of the source term is more dominant than the exponents of both damping terms. Moreover, many studies of the global well-posedness for wave systems with dissipative terms have been researched in [24-28].

Wave systems with viscoelastic terms and dissipative terms have not been fully studied. In [29] the following coupled nonlinear wave equations with dispersive terms, viscoelastic dissipative terms, and nonlinear weak damping terms are considered:

$$
\begin{aligned}
u_{t t} & -\Delta u-e \Delta u_{t t}+\int_{0}^{t} g(t-s) \Delta u(s) d s+\left|u_{t}\right|^{m-1} u_{t} \\
& =f_{1}(u, v) \\
v_{t t} & -\Delta v-f \Delta v_{t t}+\int_{0}^{t} h(t-s) \Delta v(s) d s+\left|u_{t}\right|^{r-1} v_{t} \\
& =f_{2}(u, v) .
\end{aligned}
$$

A global nonexistence theorem for certain solutions with positive initial energy is proved. Reference [30] further considered a fourth-order wave system similar to (10). In that case, the energy increases exponentially when time goes to infinity and the initial data are large enough.

Recently [31] considered a system of two coupled wave equations with dispersive and strong dissipative terms under Dirichlet boundary conditions:

$$
\begin{gathered}
\left|u_{t}\right|^{j} u_{t t}-\Delta u-\Delta u_{t t}-\Delta u_{t}=f_{1}(u, v), \\
\left|v_{t}\right|^{j} v_{t t}-\Delta v-\Delta v_{t t}-\Delta v_{t}=f_{2}(u, v),
\end{gathered}
$$

where

$$
\begin{aligned}
f_{1}(u, v) & =-a|u+v|^{r-2}(u+v)-b|u|^{r / 2-2}|v|^{r / 2} u, \\
f_{2}(u, v) & =-a|u+v|^{r-2}(u+v)-b|v|^{r / 2-2}|u|^{r / 2} v, \\
r & >2 \quad \text { if } n=1,2 ; \\
2 & <r \leq \frac{2(n-1)}{n-2} \text { if } n \geq 3 .
\end{aligned}
$$

The global existence of weak solutions and uniform decay rates (exponential one) of the solution energy were established.

Many researches considered the initial boundary value problem with global existence and blow-up of solutions for the nonlinear wave equations as follows:

$$
\begin{aligned}
& u_{t t}-\Delta u+\int_{0}^{t} g_{1}(t-s) \Delta u d s+g\left(u_{t}\right)=f_{1}(u, v) \\
& \text { in } \Omega \times(0,+\infty),
\end{aligned}
$$




$$
\begin{array}{r}
v_{t t}-\Delta v+\int_{0}^{t} g_{2}(t-s) \Delta u d s+g\left(v_{t}\right)=f_{2}(u, v) \\
\text { in } \Omega \times(0,+\infty),
\end{array}
$$

where $\Omega$ is a bounded domain in $\mathbb{R}^{n}(n=1,2,3)$ with a smooth boundary $\partial \Omega$. When the viscoelastic terms $g_{i}(i=$ 1,2 ) are absent in (13), [20] showed local and global existences of a weak solution that any weak solution blows up in finite time with negative initial energy as the same way used in [22]. Later, Said-Houari extended this blow-up result to positive initial energy. At the same time, Liu [32] studied the following Cauchy problem for the coupled system of nonlinear KleinGordon equations with damping terms:

$$
\begin{array}{cc}
u_{t t}-\Delta u+u+\gamma_{1} u_{t}=|v|^{r}|u|^{r-2} & \text { in } \mathbb{R}^{n} \times(0,+\infty), \\
v_{t t}-\Delta v+v+\gamma_{1} v_{t}=|u|^{r}|v|^{r-2} & \text { in } \mathbb{R}^{n} \times(0,+\infty),
\end{array}
$$

and the existence of standing wave with ground state was stated and a sharp criterion for global existence and blowup of solutions when $E(0)<d$ was established. The author introduced a family of potential wells and the invariant sets and vacuum isolating behavior of solutions for $0<E(0)<$ $d$ and $E(0) \leq 0$, respectively. Furthermore, he proved the global existence and asymptotic behavior of solutions when the condition is $0<E(0)<d$. Finally, a blow-up result with arbitrarily positive initial energy was obtained.

Conversely, in the presence of the memory term $\left(g_{i} \neq\right.$ $0, i=1,2$ ), some results related to the asymptotic behavior and blow-up of solutions of viscoelastic systems were discussed. For example, [33] studied problem (13) with $g\left(u_{t}\right)=$ $-\Delta u_{t}$ and $g\left(v_{t}\right)=-\Delta v_{t}$ and obtained the fact that the decay rate of the energy function is exponential under suitable conditions on the functions $g_{i}, f_{i}, i=1,2$, certain initial data in the stable set. On the contrary, when the initial data is in the unstable set, the solutions blow up in finite time under positive initial energy. For $g\left(u_{t}\right)=\left|u_{t}\right|^{m-1} u_{t}$ and $g\left(v_{t}\right)=\left|v_{t}\right|^{r-1} v_{t}$, [26] established local and global existence as well as finite time blow-up (the initial energy $E(0)<0$ ). The latter blowup result has been improved by [29] by considering a class of positive initial data. On the other hand, Messaoudi and Tatar [34] considered the following similar wave equations without considering coupling coefficient of $\Delta u$ and $\Delta v$ :

$$
\begin{gathered}
u_{t t}-\Delta u+\int_{0}^{t} g_{1}(t-s) \Delta u d s+f_{1}(u, v)=0 \\
\text { in } \Omega \times(0,+\infty), \\
v_{t t}-\Delta v+\int_{0}^{t} g_{2}(t-s) \Delta u d s+f_{2}(u, v)=0 \\
\text { in } \Omega \times(0,+\infty),
\end{gathered}
$$

where the functions $f_{1}$ and $f_{2}$ satisfy the following assumptions:

$$
\begin{aligned}
& f(u, v) \leq d\left(|u|^{\beta_{1}}+|v|^{\beta_{2}}\right), \\
& h(u, v) \leq d\left(|u|^{\beta_{3}}+|v|^{\beta_{4}}\right),
\end{aligned}
$$

and for some constant $d>0$ and $1 \leq \beta_{i} \leq n /(n-2), i=$ $1,2,3,4$, the solution goes to zero with an exponential or polynomial rate which depending on the decay rate of the relaxation functions $g_{i}, i=1,2$ was obtained. This result was improved in [35] to weaker conditions on the relaxation functions and more general coupling functions.

Additionally, Liu and Wang [36] considered the following nonlinear hyperbolic systems with damping and source terms

$$
\begin{array}{r}
u_{t t}-\left(a+b\|\nabla u\|^{2}+b\|\nabla v\|^{2}\right) \Delta u+g\left(u_{t}\right)=f(u), \\
\text { in } \Omega \times(0, T], \\
v_{t t}-\left(a+b\|\nabla u\|^{2}+b\|\nabla v\|^{2}\right) \Delta v+g\left(v_{t}\right)=h(v), \\
\text { in } \Omega \times(0, T] .
\end{array}
$$

The author defined potential well and the outer manifold of the potential well associated with system (17) and got the global existence in the case of $E(0)<d_{1} / 2$ and discussed the global nonexistence of solutions for problem (1)-(5) in the case of $E(0) \leq 0$ and $E(0)<C_{\alpha}$ on page 88 of [36]. In [37], (1)-(5) was considered with $M \equiv 1$ and without imposing the memory terms $(g=h=0)$. The rate of decay of the exponential or polynomial energy of the damping terms was obtained.

Recently, Wu [38] considered the initial boundary value problem for system (1)-(5) with $M(t)=m_{0}+\alpha\left(\|\nabla u\|^{2}+\right.$ $\left.\|\nabla v\|^{2}\right)^{\gamma}$. The assumptions for problem (1)-(5) are as follows:

(A1) The nonlinear source terms $f_{1}(u, v), f_{2}(u, v)$ satisfy

$$
\begin{aligned}
& f_{1}= \frac{\partial F}{\partial u}=(m+1) \\
& \cdot\left(a|u+v|^{m-1}(u+v)+b|u|^{(m-3) / 2}|v|^{(m+1) / 2} u\right), \\
& f_{2}= \frac{\partial F}{\partial v}=(m+1) \\
& \cdot\left(a|u+v|^{m-1}(u+v)+b|v|^{(m-3) / 2}|u|^{(m+1) / 2} v\right),
\end{aligned}
$$

where $F(u, v)=a|u+v|^{m+1}+2 b|u v|^{(m+1) / 2}$ with $a, b>$ 0 .

(A2) $M(s)$ is a nonnegative $C^{1}$ function for $s \geq 0$ satisfying

$$
M(s)=m_{0}+\alpha\left(\|\nabla u\|^{2}+\|\nabla v\|^{2}\right)^{\gamma}
$$

$$
m_{0} \geq m_{1}, \alpha \geq 0, \gamma>0 \text {. }
$$

(A3) The nonlinearity of $m, p, q$ satisfies

$$
\begin{gathered}
m>1, \quad \text { if } n=1,2 \\
\text { or } 1<m \leq 3, \quad \text { if } n=3, \\
p, q \geq 1, \quad \text { if } n=1,2, \\
\text { or } 1<p, q \leq 5, \quad \text { if } n=3 .
\end{gathered}
$$


(A4) For $s \geq 0$ the relaxation functions $g_{1}$ and $g_{2}$ are of class $C^{1}$ and satisfy

$$
\begin{aligned}
g_{1}(s) & \geq 0, \\
m_{0}-\int_{0}^{\infty} g_{1}(s) d s & =l>0, \\
g_{2}(s) & \geq 0, \\
m_{0}-\int_{0}^{\infty} g_{2}(s) d s & =k>0, \\
g_{1}^{\prime}(s) & \leq 0, \\
g_{2}^{\prime}(s) & \leq 0 .
\end{aligned}
$$

The solutions are global in time when the functions $g_{1}, g_{2}$, and $f_{i}, i=1,2$, satisfy suitable conditions and certain initial data is in the stable set. The author established the rate of decay of solutions by a difference inequality given by Nakao [39] and intended to study the blow-up phenomena of problem (1)-(5). The blow-up of solutions when the energy is negative or subcritical case was proved by adopting and modifying the methods used in [29]. In this way, the above results in [38] allowed a bigger region for the blow-up results and improved the results of Messaoudi and Said-Houari [29]. More specifically, the decay result in [38] extends the one in $[16,37]$ to problem (1)-(5), where $M$ is not a constant function and the equations considered in [38] have more dissipations.

1.2. Unsolved Problems. It is well known that in the absence of the nonlinear source term the damping term ensures global existence. In addition, without the dissipative term, the nonlinear source term causes finite time blow-up of solution. Moreover, the viscoelastic materials possess a capacity of storage and dissipation of mechanical energy; therefore, it is interesting to investigate the well-posedness of solution for the viscoelastic equation with dissipative term and nonlinear source term.

We can see that problem (1)-(5) contains system (9) $(M \equiv$ $\left.1, g_{1}=g_{2}=0\right)$, systems (10), (13), and (15) $(M \equiv 1)$, system (11) $\left(j=0, M \equiv 1, g_{1}=g_{2}=0\right)$, and system (17) $(\gamma=$ $\left.1, M \equiv 1, g_{1}=g_{2}=0\right)$ as special cases. In fact, the viscoelastic terms change the frame of the equations comparing without those viscoelastic conditions, which makes the structure of the equations and solutions more complex and which also makes energy decay faster. So the classical methods can not be applied to investigate properties of solutions. Therefore, much less is known for (1)-(5) with viscoelastic terms. We can note that in all of the above studies except [38] only the global well-posedness of solutions was proved in a relatively rough variational framework, but the global existence and finite time blow-up for problem (1)-(5) at the other initial energy levels have not been discussed yet. The global existence and blow-up results in [38] are under the assumption of negative initial energy $E(0)<0$ or $E(0)<$ $E_{1}=((m-1) / 2(m+1))(1 / \eta(m+1))^{2 /(m-1)}$. In other words, to our knowledge there are no results on global well-posedness of solutions to the initial boundary value problem for a couple of nonlinear wave equations with coupling coefficient $M(t)=m_{0}+\alpha\left(\|\nabla u\|^{2}+\|\nabla v\|^{2}\right)^{\gamma}$ of $\Delta u$ and $\Delta v$, viscoelastic terms $\int_{0}^{t} g_{1}(t-s) \Delta u d s, \int_{0}^{t} g_{2}(t-s) \Delta u d s$, and nonlinear weak dissipative terms $\left|u_{t}\right|^{k-1} u_{t},\left|v_{t}\right|^{r-1} v_{t}$. It is natural to ask a question of how the solution behaves for problem (1)-(5), which is what we want to deal with in this paper. Moreover, regarding the initial energy level, the present paper is also a comprehensive study for low energy case and high energy situation. To our knowledge this is the first try to consider this problem. The most attractive one is that we attain a blow-up result with arbitrary positive initial energy for a wave system of Kirchhoff type.

By reviewing above known results and also [19-38], we will face the fact that the following unsolved problems arise naturally. Firstly, from [38] we know the global existence for the definitely positive energy, but we know less for the initial energy which may be negative. Secondly, for general initial energy, which means the initial energy is not necessarily definitely positive, what will happen for the solution when the initial energy $E(0)<d$ or $E(0)>d$ ?

We restricts our attention to considering the global existence and blow-up at two different initial energy levels. Since the initial energy level plays a crucial role in dealing with the well-posedness of problem (1)-(5), the two cases are, respectively, tackled with different tools. For the subcritical case $E(0)<d$, there have been many tools tackling the hyperbolic problem without viscoelastic terms in $[16,17]$. We may refer the tools to deal with (1)-(5) with viscoelastic terms. By the well-known works [40-44], we see that the supercritical case $E(0)>d$ is not easy to deal with. Filippo and Marco [45] made the initial attempt to consider the global wellposedness of hyperbolic problem at high initial energy level $u_{t t}-\Delta u-\omega \Delta u_{t}+\mu u_{t}=|u|^{p-2} u$. However, they focused on particular source term $|u|^{p-1} u$, and our work is on the viscoelastic terms condition and the complex source term $f_{1}(u, v)$, $f_{2}(u, v)$. Based on the general comparison principle in [9], we try to resolve the above open problems with variational methods. In this paper, we consider the initial boundary value problem for system (10) with $e=f=0$ and the nonlinear source terms, coupling coefficient $M(s)$, the nonlinearity of $m, p, q$, and the relaxation functions $g_{1}$ and $g_{2}$ satisfying the assumptions (A1)-(A4), respectively. In addition, we consider nonlinear damping terms of the form $\left|u_{t}\right|^{k-1} u_{t}$ and $\left|v_{t}\right|^{r-1} v_{t}$ as in the first equation and in the second equation of (10), respectively.

1.3. The Main Results and Organization of the Paper. In this paper, we mainly discuss the following problems.

(1) Case $E(0)<d$ : different from the method applied in [38], we introduce a family of potential wells to obtain the results: invariant sets, global existence, and finite time blow-up.

(2) Case $E(0)>d$ : we obtain the finite time blow-up of solutions for problem (1)-(5) whose initial data have arbitrarily high initial energy.

We can summarize our main conclusions in Table 1 and use the question mark "?" to indicate the open problem. 
TABLE 1: Main results.

\begin{tabular}{lcc}
\hline & $\begin{array}{c}\text { Main results } \\
\text { Initial data }\end{array}$ & Global existence \\
\hline$E(0)<d$ & Theorem 10 & Theorem 14 \\
$E(0)>d$ & $?$ & Theorem 18 \\
\hline
\end{tabular}

The organization of the paper is as follows.

In Section 2, we introduce some notations, assumptions, and preliminaries.

From Sections 3-5, we prove the main results.

\section{Notations and Primary Lemmas}

In this section, we shall give some lemmas and some notations which will be used throughout this work. We use the standard Lebesgue space $L^{p}(\Omega)$ and Sobolev space $H_{0}^{1}(\Omega)$ with their usual norms and products as follows:

$$
\begin{aligned}
\|u\|_{L^{p}(\Omega)} & =\|u\|_{p}, \\
\|u\|_{L^{2}(\Omega)} & =\|u\|, \\
(u, v) & =\int_{\Omega} u v d x, \\
m_{1} & =\max \left\{l_{1}, k_{1}\right\}, \\
l_{1} & =\int_{0}^{\infty} g_{1}(s) d s, \\
k_{1} & =\int_{0}^{\infty} g_{2}(s) d s, \\
\beta & =\left(\|\nabla u\|^{2}+\|\nabla v\|^{2}\right)^{\gamma+1}, \\
(g \circ \phi)(t) & =\int_{0}^{t} g(t-s) \int_{\Omega}|\phi(s)-\phi(t)|^{2} d x d s .
\end{aligned}
$$

We will use the embedding $H_{0}^{1}(\Omega) \hookrightarrow L^{p}(\Omega)$ for $2 \leq p \leq$ $2 n /(n-2)$, if $n \geq 3$ or $2 \leq p$, if $n=1,2$. In this case, the embedding constant is denoted by $c_{*}$; that is,

$$
\|u\|_{p} \leq c_{*}\|\nabla u\|
$$

From assumption (A1) one can easily verify that

$$
u f_{1}(u, v)+v f_{2}(u, v)=(m+1) F(u, v) .
$$

Moreover we have the following result. Note that the following conclusion (Lemma 1) was assumed throughout many papers (see [16-31, 33, 34]); however in our opinion we think this conclusion is a deduction of assumption (A1). Thus we present this conclusion as follows and similar proof can be found in [29].

Lemma 1. There exist two positive constants $c_{0}$ and $c_{1}$ such that

$$
\begin{array}{r}
c_{0}\left(|u|^{m+1}+|v|^{m+1}\right) \leq F(u, v) \leq c_{1}\left(|u|^{m+1}+|v|^{m+1}\right), \\
\forall(u, v) \in R^{2} .
\end{array}
$$

Proof. We can see that taking $c_{1}=2^{m} a+b$ then the right-hand side of inequality (26) is trivial. For the left-hand side, the result is also trivial if $u=v=0$. If, without loss of generality, $v \neq 0$, then either $|u| \leq|v|$ or $|u|>|v|$.

For $|u| \leq|v|$, we have

$$
F(u, v)=|v|^{m+1}\left(a\left|1+\frac{u}{v}\right|^{m+1}+2 b\left|\frac{u}{v}\right|^{(m+1) / 2}\right) .
$$

Consider the continuous function

$$
j(s)=a|1+s|^{m+1}+2 b|s|^{(m+1) / 2}, \quad s \in[-1,1] .
$$

So $\min j(s) \geq 0$. If $\min j(s)=0$ then, for some $s_{0} \in[-1,1]$, we have

$$
j\left(s_{0}\right)=a\left|1+s_{0}\right|^{m+1}+2 b\left|s_{0}\right|^{(m+1) / 2}=0 .
$$

This implies that $\left|1+s_{0}\right|=\left|s_{0}\right|=0$, which is impossible. Thus $\min j(s)=2 c_{0}>0$. Therefore

$$
F(u, v) \geq 2 c_{0}|v|^{m+1} \geq 2 c_{0}|u|^{m+1} .
$$

Consequently,

$$
2 F(u, v) \geq 2 c_{0}\left(|v|^{m+1}+|u|^{m+1}\right),
$$

and then

$$
F(u, v) \geq c_{0}\left(|v|^{m+1}+|u|^{m+1}\right) .
$$

If $|u| \geq|v|$, similarly we have

$$
F(u, v) \geq c_{0}\left(|u|^{m+1}+|v|^{m+1}\right) .
$$

This leads to the desired result and completes the proof of Lemma 1.

As in [29], we still have the following results.

Lemma 2 (see [29]). Suppose that (20) holds. Then there exists a positive constant $\eta>0$ such that, for any $(u, v) \in H_{0}^{1}(\Omega) \times$ $H_{0}^{1}(\Omega)$, one has

$$
\begin{aligned}
\| u & +v\left\|^{m+1}+2\right\| u v \|_{(m+1) / 2}^{(m+1) / 2} \\
& \leq \eta\left(l\|\nabla u\|^{2}+k\|\nabla v\|^{2}\right)^{(m+1) / 2} .
\end{aligned}
$$

We also need the following technical lemma in the course of the investigation.

Lemma 3 (see [29]). For any $g_{1} \in C^{1}$ and $\phi \in H^{1}(0, T)$, one has

$$
\begin{aligned}
- & 2 \int_{0}^{t} \int_{\Omega} g(t-s) \phi \phi_{t} d x d s \\
= & \frac{d}{d t}\left((g \circ \phi)(t)-\int_{0}^{t} g(s) d s\|\phi\|^{2}\right)+g(t)\|\phi\|^{2} \\
& -\left(g^{\prime} \circ \phi\right)(t)
\end{aligned}
$$

where $(g \circ \phi)(t)=\int_{0}^{t} g(t-s) \int_{\Omega}|\phi(s)-\phi(t)|^{2} d x d s$. 
Now, we are in a position to state the local existence result to problem (1)-(5), which can be established by combining arguments of $[15,17,20,26]$.

Theorem 4 (local existence). Let $\left(u_{0}, v_{0}\right) \in H_{0}^{1}(\Omega) \times H_{0}^{1}(\Omega)$ and $\left(u_{1}, v_{1}\right) \in L^{2}(\Omega) \times L^{2}(\Omega)$ be given. Assume that (A2)-(A4) are satisfied. Then there exists a couple solution $(u, v)$ of problem (1)-(5) such that

$$
\begin{gathered}
u, v \in C\left([0, T], H^{2}(\Omega) \times H_{0}^{1}(\Omega)\right), \\
u_{t} \in C\left([0, T], H_{0}^{1}(\Omega)\right) \cap L^{p+1}(\Omega), \\
v_{t} \in C\left([0, T], H_{0}^{1}(\Omega)\right) \cap L^{q+1}(\Omega),
\end{gathered}
$$

for some $T>0$.

Remark 5 (see [46]). Condition (A1) is necessary to guarantee the hyperbolicity of the equation in (1) and (2) and condition (21) is needed to establish the local existence result.

Next for problem (1)-(5) we introduce potential energy functional:

$$
\begin{aligned}
E(t) \equiv & E(u, v) \\
= & \frac{1}{2}\left\|u_{t}\right\|^{2}+\frac{1}{2}\left\|v_{t}\right\|^{2}+\frac{1}{2}\left(m_{0}-l_{1}\right)\|\nabla u\|^{2} \\
& +\frac{1}{2}\left(m_{0}-k_{1}\right)\|\nabla v\|^{2}+\frac{\alpha \beta}{2(\gamma+1)} \\
& +\frac{1}{2}\left(g_{1} \circ \nabla u\right)(t)+\frac{1}{2}\left(g_{2} \circ \nabla v\right)(t) \\
& -\int_{\Omega} F(u, v) d x .
\end{aligned}
$$

Potential energy functional:

$$
\begin{aligned}
J(t) \equiv & J(u, v) \\
= & \frac{1}{2}\left(m_{0}-l_{1}\right)\|\nabla u\|^{2}+\frac{1}{2}\left(m_{0}-k_{1}\right)\|\nabla v\|^{2} \\
& +\frac{\alpha \beta}{2(\gamma+1)}+\frac{1}{2}\left(g_{1} \circ \nabla u\right)(t) \\
& +\frac{1}{2}\left(g_{2} \circ \nabla v\right)(t)-\int_{\Omega} F(u, v) d x .
\end{aligned}
$$

Nehari functional:

$$
\begin{aligned}
I(t) \equiv & I(u, v) \\
= & \left(m_{0}-l_{1}\right)\|\nabla u\|^{2}+\left(m_{0}-k_{1}\right)\|\nabla v\|^{2}+\alpha \beta \\
& +\left(g_{1} \circ \nabla u\right)(t)+\left(g_{2} \circ \nabla v\right)(t) \\
& -(m+1) \int_{\Omega} F(u, v) d x .
\end{aligned}
$$

For the definition of $F(u, v)$ please see assumption (A1) in the beginning of this paper. Moreover we introduce the potential well (stable set)

$$
\begin{aligned}
W= & \left\{(u, v) \in H_{0}^{1}(\Omega) \times H_{0}^{1}(\Omega) \mid I(u, v)>0\right\} \\
& \cup\{(0,0)\},
\end{aligned}
$$

and the outer space of potential well (unstable set)

$$
V=\left\{(u, v) \in H_{0}^{1}(\Omega) \times H_{0}^{1}(\Omega) \mid I(u, v)<0\right\} .
$$

Moreover we define

$$
d=\inf _{(u, v) \in H_{0}^{1}(\Omega) \times H_{0}^{1}(\Omega) \backslash\{(0,0)\}}\left(\sup _{\lambda \geq 0} J(\lambda u, \lambda v)\right),
$$

or equivalently

$$
d=\inf _{(u, v) \in \mathcal{N}} J(u, v)
$$

where $\mathcal{N}=\left\{(u, v) \in H_{0}^{1}(\Omega) \times H_{0}^{1}(\Omega) \backslash\{(0,0)\} \mid I(u, v)=0\right\}$.

Lemma 6 (depth of potential well). The depth of potential well $d=\left((m-1)\left(m_{0}-m_{1}\right) / 2(m+1)\right)\left(\left(m_{0}-m_{1}\right) / c_{1}(m+\right.$ 1) $\left.C_{*}^{m+1}\right)^{2 /(m-1)}$, where $c_{1}$ is defined in (26) and $C_{*}$ is the best imbedding constant from $H_{0}^{1}(\Omega)$ into $L^{m+1}(\Omega)$.

Proof. From the definition of $d$, we have $(u, v) \in \mathcal{N}$; that is, $I(u, v)=0$. Then on the one hand from Lemma 1 we get

$$
\begin{aligned}
& \left(m_{0}-l_{1}\right)\|\nabla u\|^{2}+\left(m_{0}-k_{1}\right)\|\nabla v\|^{2}+\alpha \beta \\
& \quad+\left(g_{1} \circ \nabla u\right)(t)+\left(g_{2} \circ \nabla v\right)(t) \\
& \quad=(m+1) \int_{\Omega} F(u, v) d x \\
& \quad \leq c_{1}(m+1)\left(\|u\|_{m+1}^{m+1}+\|v\|_{m+1}^{m+1}\right) \\
& \quad \leq c_{1}(m+1) C_{*}^{m+1}\left(\|\nabla u\|^{2}+\|\nabla v\|^{2}\right)^{(m+1) / 2} .
\end{aligned}
$$

Notice that, from assumption (A4) and the definitions $\beta$ and $m_{1}$, we have

$$
\begin{aligned}
& \left(m_{0}-m_{1}\right)\left(\|\nabla u\|^{2}+\|\nabla v\|^{2}\right) \\
& \quad \leq c_{1}(m+1) C_{*}^{m+1}\left(\|\nabla u\|^{2}+\|\nabla v\|^{2}\right)^{(m+1) / 2}
\end{aligned}
$$

that is

$$
\|\nabla u\|^{2}+\|\nabla v\|^{2} \geq\left(\frac{m_{0}-m_{1}}{c_{1}(m+1) C_{*}^{m+1}}\right)^{2 /(m-1)} .
$$


On the other hand notice $I(u, v)=0$; moreover by virtue of (38), (39), and (78), we get

$$
\begin{aligned}
& J(u, v)=\left(\frac{1}{2}-\frac{1}{m+1}\right) \\
& \cdot\left(\left(m_{0}-l_{1}\right)\|\nabla u\|^{2}+\left(m_{0}-k_{1}\right)\|\nabla v\|^{2}\right) \\
& +\left(\frac{\alpha}{2(\gamma+1)}-\frac{\alpha}{m+1}\right) \beta+\left(\frac{1}{2}-\frac{1}{m+1}\right) \\
& \cdot\left(g_{1} \circ \nabla u\right)(t)+\left(\frac{1}{2}-\frac{1}{m+1}\right)\left(g_{2} \circ \nabla v\right)(t) \\
& +\frac{1}{m+1} I(u, v) \geq\left(\frac{1}{2}-\frac{1}{m+1}\right) \\
& \cdot\left(\left(m_{0}-l_{1}\right)\|\nabla u\|^{2}+\left(m_{0}-k_{1}\right)\|\nabla v\|^{2}\right) \\
& \geq\left(\frac{1}{2}-\frac{1}{m+1}\right)\left(m_{0}-m_{1}\right)\left(\|\nabla u\|^{2}+\|\nabla v\|^{2}\right) \\
& \geq\left(\frac{1}{2}-\frac{1}{m+1}\right)\left(m_{0}-m_{1}\right) \\
& \cdot\left(\frac{m-m_{1}}{c_{1}(m+1) C_{*}^{m+1}}\right)^{2 /(m-1)},
\end{aligned}
$$

and hence we have $d=(1 / 2-1 /(m+1))\left(m_{0}-m_{1}\right)\left(\left(m_{0}-\right.\right.$ $\left.\left.m_{1}\right) / c_{1}(m+1) C_{*}^{m+1}\right)^{2 /(m-1)}$.

Lemma 7 (nonincreasing energy). Let $(u, v)$ be a solution of problem (1)-(5); then $E(t)$ is a nonincreasing function for $t \geq 0$; that is,

$$
\begin{aligned}
\frac{d}{d t} E(t)= & -\left\|u_{t}\right\|_{p+1}^{p+1}-\left\|v_{t}\right\|_{q+1}^{q+1}+\frac{1}{2}\left(g_{1}^{\prime} \circ \nabla u\right)(t) \\
& +\frac{1}{2}\left(g_{2}^{\prime} \circ \nabla v\right)(t)-\frac{1}{2} g_{1}(t)\|\nabla u\|^{2} \\
& -\frac{1}{2} g_{2}(t)\|\nabla v\|^{2} \leq 0, \quad \forall t \geq 0 .
\end{aligned}
$$

Proof. Multiplying (1) by $u_{t}$ and (2) by $v_{t}$, integrating them over $\Omega$, and then adding the results together and integrating by parts, it follows that

$$
\begin{aligned}
& \frac{d}{d t}\left(\frac { 1 } { 2 } \left(\left\|u_{t}\right\|^{2}+\left\|v_{t}\right\|^{2}+\left(\|\nabla u\|^{2}+\|\nabla v\|^{2}\right)\right.\right. \\
& \left.\left.+\frac{\alpha}{\gamma+1}\left(\|\nabla u\|^{2}+\|\nabla v\|^{2}\right)^{\gamma+1}\right)-\int_{\Omega} F(u, v) d x\right) \\
& =-\left\|u_{t}\right\|_{p+1}^{p+1}-\left\|v_{t}\right\|_{q+1}^{q+1}+\int_{0}^{t} \int_{\Omega} g_{1}(t-s) \nabla u(s) \\
& \cdot \nabla u_{t} d x d s+\int_{0}^{t} \int_{\Omega} g_{2}(t-s) \nabla v(s) \cdot \nabla v_{t} d x d s .
\end{aligned}
$$

Exploiting Lemma 3 on the third term and the fourth term on the right side of the above equality, we can obtain (48) for any regular solution.
3. Global Existence under the Case $E(0)<d$

Now we give the following definition of weak solution for problem (1)-(5).

Definition 8 (weak solution). A function $(u, v)$ is called a weak solution of problem (1)-(5) on $\Omega \times[0, T]$, if it satisfies $(u, v) \in L^{\infty}\left([0, T], H_{0}^{1}(\Omega) \times H_{0}^{1}(\Omega)\right)$ with $\left(u_{t}, v_{t}\right) \in$ $L^{\infty}\left([0, T], L^{2}(\Omega) \times L^{2}(\Omega)\right) \cap L^{\infty}\left([0, T], L^{r+1}(\Omega) \times L^{r+1}(\Omega)\right)$ and

$$
\begin{aligned}
& \left(u_{t}, \omega_{1}\right)-\int_{0}^{t}\left(\left(m_{0}+\alpha\left(\|\nabla u\|^{2}+\|\nabla v\|^{2}\right)^{\gamma}\right) \Delta u, \omega_{1}\right) d \tau \\
& \quad-\int_{0}^{t} \int_{0}^{\sigma} g_{1}(\sigma-\tau)\left(\nabla u(\tau), \nabla w_{1}\right) d \tau d \sigma \\
& \quad+\int_{0}^{t}\left(\left|u_{t}\right|^{p-1} u_{t}, \omega_{1}\right) d \tau=\int_{0}^{t}\left(f_{1}(u, v), \omega_{1}\right) d \tau \\
& +\left(u_{1}, \omega_{1}\right), \quad \forall \omega_{1} \in H_{0}^{1}(\Omega), \\
& \left(v_{t}, \omega_{2}\right)-\int_{0}^{t}\left(\left(m_{0}+\alpha\left(\|\nabla u\|^{2}+\|\nabla v\|^{2}\right)^{\gamma}\right) \Delta v, \omega_{2}\right) d \tau \\
& \quad-\int_{0}^{t} \int_{0}^{\sigma} g_{2}(\sigma-\tau)\left(\nabla u(\tau), \nabla w_{2}\right) d \tau d \sigma \\
& \quad+\int_{0}^{t}\left(\left|v_{t}\right|^{q-1} v_{t}, \omega_{2}\right) d \tau=\int_{0}^{t}\left(f_{2}(u, v), \omega_{2}\right) d \tau \\
& \quad+\left(v_{1}, \omega_{2}\right), \quad \forall \omega_{2} \in H_{0}^{1}(\Omega),
\end{aligned}
$$

with

$$
\begin{gathered}
u(0, x)=u_{0}(x), \\
u_{t}(0, x)=u_{1}(x), \\
v(0, x)=v_{0}(x), \\
v_{t}(0, x)=v_{1}(x) .
\end{gathered}
$$

Lemma 9 (invariant set $W$ ). Let $\left(u_{0}, v_{0}\right) \in H_{0}^{1}(\Omega) \times H_{0}^{1}(\Omega)$, $\left(u_{1}, v_{1}\right) \in L^{2}(\Omega) \times L^{2}(\Omega)$, and $(\boldsymbol{A 1})-(\boldsymbol{A} 4)$ hold. Then all solutions of problem (1)-(5) with $E(0)<d$ belong to $W$, provided $\left(u_{0}, v_{0}\right) \in W$.

Proof. Let $(u(t), v(t))$ be any local weak solution of problem (1)-(5) with $E(0)<d$ and $\left(u_{0}, v_{0}\right) \in W$ and $T$ be the existence time of $(u(t), v(t))$. Then it follows from Lemma 7 that $E(u(t), v(t)) \leq E(0)<d$. Thus if suffices to show that $I(u(t), v(t))>0$ for $0<t<T$. Suppose that there exists $t_{1} \in(0, T)$ such that $I\left(u\left(t_{1}\right), v\left(t_{1}\right)\right) \leq 0$. From the continuity of the solution in time, there exists $t_{*} \in(0, T)$ such that $I\left(u\left(t_{*}\right), v\left(t_{*}\right)\right)=0$. Then from the definition of $d$ we have

$$
d \leq J\left(u\left(t_{*}\right), v\left(t_{*}\right)\right) \leq E\left(u\left(t_{*}\right), v\left(t_{*}\right)\right) \leq \mathrm{E}(0)<d,
$$

which is a contradiction.

Then we give the global existence of solutions for problem (1)-(5) with low initial energy level $E(0)<d$. 
Theorem 10 (global existence when $E(0)<d$ ). Let $\left(u_{0}, v_{0}\right) \in$ $H_{0}^{1}(\Omega) \times H_{0}^{1}(\Omega),\left(u_{1}, v_{1}\right) \in L^{2}(\Omega) \times L^{2}(\Omega)$, and (A1)-(A4) hold. Assume that $E(0)<d$ and $\left(u_{0}, v_{0}\right) \in W$. Then problem (1)-(5) admits a global weak solution $u(t), v(t) \in L^{\infty}\left(0, T ; H_{0}^{1}(\Omega)\right)$, $u_{t}(t), v_{t}(t) \in L^{\infty}\left(0, T ; L^{2}(\Omega)\right)$, and $(u, v) \in W$ for $0 \leq t \leq \infty$.

Proof. Let $\left\{\omega_{j}\right\}$ be a basis in $H_{0}^{1}(\Omega)$ given by the eigenfunction of the operator $-\Delta$ and it constructs a complete orthogonal system such that $\left\|\omega_{j}\right\|=1$ for all $j$. Then $\left\{\omega_{j}\right\}$ is orthogonal and complete in $L^{2}(\Omega)$ and in $H_{0}^{1}(\Omega)$. Let $V_{m}$ be the space generated by $\left\{\omega_{1}, \omega_{2}, \ldots, \omega_{m}\right\}, m \in \mathbb{N}$. Construct the approximate solutions of problem (1)-(5):

$$
\begin{aligned}
& u_{m}(x, t)=\sum_{j=1}^{m} g_{j m}(t) w_{j}(x), \quad m=1,2, \ldots, \\
& v_{m}(x, t)=\sum_{j=1}^{m} h_{j m}(t) w_{j}(x), \quad m=1,2, \ldots,
\end{aligned}
$$

satisfying

$$
\begin{aligned}
& \left(u_{m t t}(t), \omega\right)+\left(\left(m_{0}+\alpha\left(\left\|\nabla u_{m}\right\|^{2}+\left\|\nabla v_{m}\right\|^{2}\right)^{\gamma}\right), \nabla \omega\right) \\
& \quad-\int_{0}^{t} g_{1}(t-\tau)\left(\nabla u_{m}(\tau), \nabla \omega\right) d \tau \\
& +\left(\left|u_{m t}\right|^{p-1} u_{m t}, \omega\right)=\left(f_{1}\left(u_{m t}, v_{m t}\right), \omega\right), \\
& \quad \forall \omega \in V_{m}, \\
& \left(v_{m t t}(t), \omega\right)+\left(\left(m_{0}+\alpha\left(\left\|\nabla u_{m}\right\|^{2}+\left\|\nabla v_{m}\right\|^{2}\right)^{\gamma}\right), \nabla \omega\right) \\
& \quad-\int_{0}^{t} g_{2}(t-\tau)\left(\nabla v_{m}(\tau), \nabla \omega\right) d \tau \\
& \quad+\left(\left|v_{m t}\right|^{q-1} v_{m t}, \omega\right)=\left(f_{2}\left(u_{m t}, v_{m t}\right), \omega\right),
\end{aligned}
$$$$
\forall \omega \in V_{m},
$$

$v_{m}(0)=v_{0 m}=\sum_{j=1}^{m}\left(v_{0}, \omega_{j}\right) \omega_{j} \longrightarrow v_{0} \quad$ in $H_{0}^{1}(\Omega)$,

$u_{m t}(0)=u_{1 m}=\sum_{j=1}^{m}\left(u_{1}, \omega_{j}\right) \omega_{j} \longrightarrow u_{1} \quad$ in $L^{2}(\Omega)$,

$$
v_{m t}(0)=v_{1 m}=\sum_{j=1}^{m}\left(v_{1}, \omega_{j}\right) \omega_{j} \longrightarrow v_{1} \quad \text { in } L^{2}(\Omega) .
$$

Multiplying (54) and (55) by $g_{s m}^{\prime}(t), h_{s m}^{\prime}(t)$, respectively, and summing for $s$ and adding these two equations, we can deduce

$$
\begin{aligned}
\frac{d}{d t} E\left(u_{m}(t), v_{m}(t)\right)= & \frac{1}{2}\left(g_{1}^{\prime} \circ \nabla u_{m}\right)(t)-\left\|u_{m t}\right\|_{p+1}^{p+1} \\
& -\frac{1}{2} g_{1}(t)\left\|\nabla u_{m}\right\|^{2}
\end{aligned}
$$

$$
\begin{aligned}
& +\frac{1}{2}\left(g_{2}^{\prime} \circ \nabla v_{m}\right)(t) \\
& -\frac{1}{2} g_{2}(t)\left\|\nabla v_{m}\right\|^{2}-\left\|v_{m t}\right\|_{q+1}^{q+1} .
\end{aligned}
$$

Integrating the above equation with respect to $t$, we have

$$
\begin{aligned}
& E_{m}(t)+\int_{0}^{t}\left(\left\|u_{m \tau}\right\|_{p+1}^{p+1}+\frac{1}{2} g_{1}(\tau)\left\|\nabla u_{m}\right\|^{2}\right. \\
& \left.-\frac{1}{2}\left(g_{1}^{\prime} \circ \nabla u_{m}\right)(\tau)\right) d \tau+\int_{0}^{t}\left(\left\|v_{m \tau}\right\|_{q+1}^{q+1}\right. \\
& \left.+\frac{1}{2} g_{2}(\tau)\left\|\nabla v_{m}\right\|^{2}-\frac{1}{2}\left(g_{2}^{\prime} \circ \nabla v_{m}\right)(\tau)\right) d \tau \\
& =E_{m}(0),
\end{aligned}
$$

where

$$
\begin{aligned}
E_{m}(t):= & \frac{1}{2}\left\|u_{m t}\right\|^{2}+\frac{1}{2}\left\|v_{m t}\right\|^{2}+\frac{1}{2}\left(m_{0}-l_{1}\right)\left\|\nabla u_{m}\right\|^{2} \\
& +\frac{1}{2}\left(m_{0}-k_{1}\right)\left\|\nabla v_{m}\right\|^{2}+\frac{\alpha \beta}{2(\gamma+1)} \\
& +\frac{1}{2}\left(g_{1} \circ \nabla u_{m}\right)(t)+\frac{1}{2}\left(g_{2} \circ \nabla v_{m}\right)(t) \\
& -\int_{\Omega} F\left(u_{m}, v_{m}\right) d x \\
= & \frac{1}{2}\left(\left\|u_{m t}\right\|^{2}+\left\|v_{m t}\right\|^{2}\right)+J\left(u_{m}, v_{m}\right),
\end{aligned}
$$

From $\left(u_{0}, v_{0}\right) \in H_{0}^{1}(\Omega) \times H_{0}^{1}(\Omega)$, (56), and (57) we get that as $m \rightarrow \infty$

$$
\begin{aligned}
\left\|u_{m t}(0)\right\| & \longrightarrow\left\|u_{1}\right\|, \\
\left\|v_{m t}(0)\right\| & \longrightarrow\left\|v_{1}\right\|, \\
\left\|\nabla u_{m}(0)\right\| & \longrightarrow\left\|\nabla u_{0}\right\|, \\
\left\|\nabla v_{m}(0)\right\| & \longrightarrow\left\|\nabla v_{0}\right\| .
\end{aligned}
$$

Therefore we have $E_{m}(0) \rightarrow E(0)$ as $m \rightarrow 0$. Then for sufficiently large $m$ we have

$$
\begin{aligned}
& \frac{1}{2}\left(\left\|u_{m t}\right\|^{2}+\left\|v_{m t}\right\|^{2}\right)+J\left(u_{m}, v_{m}\right)+\int_{0}^{t}\left(\left\|u_{m \tau}\right\|_{p+1}^{p+1}\right. \\
& \left.\quad+\frac{1}{2} g_{1}(\tau)\left\|\nabla u_{m}\right\|^{2}-\frac{1}{2}\left(g_{1}^{\prime} \circ \nabla u_{m}\right)(\tau)\right) d \tau \\
& \quad+\int_{0}^{t}\left(\left\|v_{m \tau}\right\|_{q+1}^{q+1}+\frac{1}{2} g_{2}(\tau)\left\|\nabla v_{m}\right\|^{2}\right. \\
& \left.\quad-\frac{1}{2}\left(g_{2}^{\prime} \circ \nabla v_{m}\right)(\tau)\right) d \tau<d .
\end{aligned}
$$


Note that

$$
\begin{aligned}
J(u, v)= & \left(\frac{1}{2}-\frac{1}{m+1}\right) \\
& \cdot\left(\left(m_{0}-l_{1}\right)\|\nabla u\|^{2}+\left(m_{0}-k_{1}\right)\|\nabla v\|^{2}\right) \\
& +\left(\frac{\alpha}{2(\gamma+1)}-\frac{\alpha}{m+1}\right) \beta+\left(\frac{1}{2}-\frac{1}{m+1}\right) \\
& \cdot\left(g_{1} \circ \nabla u\right)(t)+\left(\frac{1}{2}-\frac{1}{m+1}\right)\left(g_{2} \circ \nabla v\right)(t) \\
& +\frac{1}{m+1} I(u, v) .
\end{aligned}
$$

Hence, from (62) and (63), we get

$$
\begin{aligned}
& \frac{1}{2}\left(\left\|u_{m t}\right\|^{2}+\left\|v_{m t}\right\|^{2}\right)+\left(\frac{\alpha}{2(\gamma+1)}-\frac{\alpha}{m+1}\right) \beta \\
& \quad+\frac{1}{m+1} I\left(u_{m}, v_{m}\right)+\left(\frac{1}{2}-\frac{1}{m+1}\right) \\
& \quad \cdot\left(\left(m_{0}-l_{1}\right)\left\|\nabla u_{m}\right\|^{2}+\left(m_{0}-k_{1}\right)\left\|\nabla v_{m}\right\|^{2}\right) \\
& \quad+\left(\frac{1}{2}-\frac{1}{m+1}\right)\left(g_{1} \circ \nabla u_{m}\right)(t)+\left(\frac{1}{2}-\frac{1}{m+1}\right) \\
& \quad \cdot\left(g_{2} \circ \nabla v_{m}\right)(t)<d .
\end{aligned}
$$

By $\left(u_{0}, v_{0}\right) \in W$ and

$$
\begin{aligned}
& \frac{1}{2}\left(\left\|u_{m t}(0)\right\|^{2}+\left\|v_{m t}(0)\right\|^{2}\right)+J\left(u_{m}(0), v_{m}(0)\right) \\
& \quad=E_{m}(0)
\end{aligned}
$$

taking into account (56) and (57), we can get $\left(u_{m}(0), v_{m}(0)\right) \epsilon$ $W$ for sufficiently large $m$. From (62) and an argument similar to the proof of Lemma 9 we can prove that $\left(u_{m}(t), v_{m}(t)\right) \in W$ for $0 \leq t<\infty$ and sufficiently large $m$. Thus (64) gives

$$
\begin{aligned}
& \frac{1}{2}\left(\left\|u_{m t}\right\|^{2}+\left\|v_{m t}\right\|^{2}\right)+\left(\frac{\alpha}{2(\gamma+1)}-\frac{\alpha}{m+1}\right) \beta \\
& \quad+\left(\frac{1}{2}-\frac{1}{m+1}\right) \\
& +\left(\left(m_{0}-l_{1}\right)\left\|\nabla u_{m}\right\|^{2}+\left(m_{0}-k_{1}\right)\left\|\nabla v_{m}\right\|^{2}\right) \\
& +\left(\frac{1}{2}-\frac{1}{m+1}\right)\left(g_{1} \circ \nabla u_{m}\right)(t)+\left(\frac{1}{2}-\frac{1}{m+1}\right) \\
& \quad \cdot\left(g_{2} \circ \nabla v_{m}\right)(t)<d,
\end{aligned}
$$

for sufficiently large $m$ and $t \in[0, \infty)$. Inequality (66) gives

$u_{m}$ and $v_{m}$ are both bounded in $L^{\infty}\left(0, \infty ; H_{0}^{1}(\Omega)\right)$,

$u_{m t}$ and $v_{m t}$ are both bounded in $L^{\infty}(0, \infty$;

$$
\left.L^{2}(\Omega)\right)
$$

Furthermore, according to (68), the following results hold:

$\left|u_{m t}\right|^{p-1} u_{m t}$ is bounded in $L^{\infty}\left(0, \infty ; L^{r}(\Omega)\right)$,

$$
\text { where } r=\frac{p+1}{p}
$$

$\left|v_{m t}\right|^{q-1} v_{m t}$ is bounded in $L^{\infty}\left(0, \infty ; L^{r}(\Omega)\right)$,

$$
\text { where } r=\frac{q+1}{q} \text {, }
$$

$$
\left|u_{m}\right|^{m-1} u_{m} \text { and }\left|v_{m}\right|^{m-1}
$$

- $v_{m}$ are both bounded in $L^{\infty}\left(0, \infty ; L^{r}(\Omega)\right)$,

$$
\text { where } r=\frac{m+1}{m} \text {. }
$$

Hence integrating (54) and (55) with respect to $t$, for every $s \in H_{0}^{1}(\Omega)$ and $0 \leq t<\infty$, we have

$$
\begin{aligned}
& \left(u_{m t}, w_{s}\right) \\
& \quad-\int_{0}^{t}\left(\left(m_{0}+\alpha\left(\left\|\nabla u_{m}\right\|^{2}+\left\|\nabla v_{m}\right\|^{2}\right)^{\gamma}\right) \Delta u_{m}, w_{s}\right) d \tau \\
& \quad-\int_{0}^{t} \int_{0}^{\sigma} g_{1}(\sigma-\tau)\left(\nabla u_{m}(\tau), \nabla w_{s}\right) d \tau d \sigma \\
& +\int_{0}^{t}\left(\left|u_{m t}\right|^{p-1} u_{m t}, w_{s}\right) d \tau \\
& \quad=\int_{0}^{t}\left(f_{1}\left(u_{m}, v_{m}\right), w_{s}\right) d \tau+\left(u_{1}, w_{s}\right), \\
& \left(v_{m t}, w_{s}\right) \\
& \quad-\int_{0}^{t}\left(\left(m_{0}+\alpha\left(\left\|\nabla u_{m}\right\|^{2}+\left\|\nabla v_{m}\right\|^{2}\right)^{\gamma}\right) \Delta v_{m}, w_{s}\right) d \tau \\
& \quad-\int_{0}^{t} \int_{0}^{\sigma} g_{2}(\sigma-\tau)\left(\nabla u_{m}(\tau), \nabla w_{s}\right) d \tau d \sigma \\
& \quad+\int_{0}^{t}\left(\left|v_{m t}\right|^{q-1} v_{m t}, w_{s}\right) d \tau \\
& \quad=\int_{0}^{t}\left(f_{2}\left(u_{m}, v_{m}\right), w_{s}\right) d \tau+\left(v_{1}, w_{s}\right) .
\end{aligned}
$$

Therefore, up to a subsequence, by (67)-(71), we may pass to the limit in (72) and obtain a weak solution $(u, v)$ of problem (1)-(5) with the above regularity (67)-(71) and (50). On the other hand, from $(56)$ and (57) we have $(u(x, 0), v(x, 0))=$ $\left(u_{0}(x), v_{0}(x)\right)$ in $H_{0}^{1}(\Omega) \times H_{0}^{1}(\Omega)$ and $\left(u_{t}(x, 0), v_{t}(x, 0)\right)=$ $\left(u_{1}(x), v_{1}(x)\right)$ in $L^{2}(\Omega) \times L^{2}(\Omega)$.

\section{Finite Time Blow-Up When $E(0)<d$}

Let us turn to discuss blow-up properties of solutions for system (1)-(5) when $E(0)<d, g\left(u_{t}\right)=u_{t}, g\left(v_{t}\right)=v_{t}$. We firstly give the following definition of finite time blow-up of weak solution for problem (1)-(5). 
Definition 11 (finite time blow-up). A solution $(u, v)$ of problem (1)-(5) is called a blow-up solution if there exists a finite time $T$ such that

$$
\limsup _{t \rightarrow T^{-}} \int_{\Omega}\left(u^{2}+v^{2}\right) d x=\infty .
$$

By the same argument as Lemma 9, we can get the following lemma.

Lemma 12 (invariant set $V)$. Let $\left(u_{0}, v_{0}\right) \in H_{0}^{1}(\Omega) \times H_{0}^{1}(\Omega)$, $\left(u_{1}, v_{1}\right) \in L^{2}(\Omega) \times L^{2}(\Omega)$, and $(\boldsymbol{A 1})-(\boldsymbol{A} 4)$ hold. Then all solutions of problem (1)-(5) with $E(0)<d$ belong to $V$, provided $\left(u_{0}, v_{0}\right) \in V$.

In order to prove Theorem 14 we state some relations of the depth of potential well $d$, norm $\|\nabla u\|^{2}+\|\nabla v\|^{2}$, and function $F(u, v)$ as follows.

Lemma 13. Under the assumptions of Lemma 15, one has

$$
d<\frac{(m-1)\left(m_{0}-m_{1}\right)}{2(m+1)}\left(\|\nabla u\|^{2}+\|\nabla v\|^{2}\right) .
$$

Proof. From Lemma 6 for the depth of potential well $d$, we have

$$
d=\frac{(m-1)\left(m_{0}-m_{1}\right)}{2(m+1)}\left(\frac{m_{0}-m_{1}}{c_{1}(m+1) C_{*}^{m+1}}\right)^{2 /(m-1)},
$$

where $c_{1}$ is defined in (26) and $C_{*}$ is the best imbedding constant from $H_{0}^{1}(\Omega)$ into $L^{m+1}(\Omega)$. By Lemma 15, we get $(u, v) \in V$; that is, $I(u, v)<0$. Moreover by Lemma 1 and Sobolev embedding inequality, $I(u, v)<0$ implies that

$$
\begin{aligned}
& \left(m_{0}-l_{1}\right)\|\nabla u\|^{2}+\left(m_{0}-k_{1}\right)\|\nabla v\|^{2}+\alpha \beta \\
& \quad+\left(g_{1} \circ \nabla u\right)(t)+\left(g_{2} \circ \nabla v\right)(t) \\
& \quad<(m+1) \int_{\Omega} F(u, v) d x \\
& \quad \leq c_{1}(m+1)\left(\|u\|_{m+1}^{m+1}+\|v\|_{m+1}^{m+1}\right) \\
& \quad \leq c_{1}(m+1) C_{*}^{m+1}\left(\|\nabla u\|^{2}+\|\nabla v\|^{2}\right)^{(m+1) / 2} .
\end{aligned}
$$

Notice that, from assumption (A4) and the definitions $\beta$ and $m_{1}$, we have

$$
\begin{aligned}
& \left(m_{0}-m_{1}\right)\left(\|\nabla u\|^{2}+\|\nabla v\|^{2}\right) \\
& \quad<c_{1}(m+1) C_{*}^{m+1}\left(\|\nabla u\|^{2}+\|\nabla v\|^{2}\right)^{(m+1) / 2} ;
\end{aligned}
$$

that is

$$
\|\nabla u\|^{2}+\|\nabla v\|^{2}>\left(\frac{m_{0}-m_{1}}{c_{1}(m+1) C_{*}^{m+1}}\right)^{2 /(m-1)} .
$$

Hence we obtain

$$
d<\frac{(m-1)\left(m_{0}-m_{1}\right)}{2(m+1)}\left(\|\nabla u\|^{2}+\|\nabla v\|^{2}\right) .
$$

A finite time blow-up result of solutions for problem (1)-(5) is showed as follows.

Theorem 14 (finite time blow-up when $E(0)<d$ ). Let $\left(u_{0}, v_{0}\right) \in H_{0}^{1}(\Omega) \times H_{0}^{1}(\Omega),\left(u_{1}, v_{1}\right) \in L^{2}(\Omega) \times L^{2}(\Omega)$, and (A1)-(A4) hold; $1<p<m, 1<q<m$ hold. Assume that $E(0)<\zeta d(\zeta<1),\left(u_{0}, v_{0}\right) \in V$, and $m$ satisfy

$$
m_{1} \leq \frac{(m-1)(1-\zeta) m_{0}}{(m-1)(1-\zeta)+1 /(m+1)}
$$

Then the existence time of solution of problem (1)-(5) is finite.

Proof. Let $(u, v)$ be any solution of problem (1)-(5) with $E(0)<d$ and $\left(u_{0}, v_{0}\right) \in V$. Next, we prove the solution of problem (1)-(5) blows up in finite time. Suppose by contradiction that the solution $(u(t), v(t))$ is global. Then, for any $T_{0}>0$, we define a auxiliary function $F(t)$ by

$$
\begin{aligned}
F(t)= & \|u\|^{2}+\|v\|^{2}+\int_{0}^{t}\left(\|u\|^{2}+\|v\|^{2}\right) d \tau \\
& +\left(T_{0}-t\right)\left(\left\|u_{0}\right\|^{2}+\left\|v_{0}\right\|^{2}\right)
\end{aligned}
$$

Clearly $F(t)>0$ for all $t \in\left[0, T_{0}\right]$. From the continuity of $F(t)$ in $t$, it is easy to see that there exists $\rho>0$ (independent of the choice of $T_{0}$ ) such that

$$
F(t) \geq \rho, \quad \forall t \in\left[0, T_{0}\right] .
$$

Then for $t \in\left[0, T_{0}\right]$ we have

$$
\begin{aligned}
F^{\prime}(t)= & 2\left(u, u_{t}\right)+2\left(v, v_{t}\right)-\left(\left\|u_{0}\right\|^{2}+\left\|v_{0}\right\|^{2}\right) \\
& +\left(\|u\|^{2}+\|v\|^{2}\right) \\
= & 2\left(u, u_{t}\right)+2\left(v, v_{t}\right) \\
& +2 \int_{0}^{t}\left(\left(u(\tau), u_{\tau}(\tau)\right)+\left(v(\tau), v_{\tau}(\tau)\right)\right) d \tau \\
F^{\prime \prime}(t)= & 2\left(\left\|u_{t}\right\|^{2}+\left\|v_{t}\right\|^{2}\right)+2\left(u, u_{t t}\right)+2\left(v, v_{t t}\right) \\
& +2\left(u, u_{t}\right)+2\left(v, v_{t}\right) \\
= & 2\left(\left\|u_{t}\right\|^{2}+\left\|v_{t}\right\|^{2}\right) \\
& -2\left(m_{0}+\alpha\left(\|\nabla u\|^{2}+\|\nabla v\|^{2}\right)^{\gamma}\right)\|\nabla u\|^{2} \\
& +2 \int_{0}^{t} g_{1}(t-\tau) \int_{\Omega} \nabla u(t) \nabla u(\tau) d x d \tau \\
& +2\left(f_{1}(u, v), u\right) \\
& +2\left(m_{0}+\alpha\left(\|\nabla u\|^{2}+\|\nabla v\|^{2}\right)^{\gamma}\right)\|\nabla v\|^{2} \\
& +2 \int_{0}^{t} g_{2}(t-\tau) \int_{\Omega} \nabla v(t) \nabla v(\tau) d x d \tau \\
& 2\left(f_{2}(u, v), v\right) .
\end{aligned}
$$


Applying Young's inequality to estimate the fourth term on the right side of (84), we have

$$
\begin{aligned}
& 2 \int_{0}^{t} g_{1}(t-\tau) \int_{\Omega} \nabla u(t) \nabla u(\tau) d x d \tau \\
& =2 \int_{0}^{t} g_{1}(t-\tau)\|\nabla u(t)\|^{2} d \tau \\
& \quad+2 \int_{0}^{t} g_{1}(t-\tau) \int_{\Omega} \nabla u(t)(\nabla u(\tau)-\nabla u(t)) d x d \tau \\
& \geq 2 \int_{0}^{t} g_{1}(t-\tau)\|\nabla u(t)\|^{2} d \tau \\
& \quad-2 \int_{0}^{t} g_{1}(t-\tau)\|\nabla u(t)\|\|\nabla u(\tau)-\nabla u(t)\| d \tau \\
& \geq 2 \int_{0}^{t} g_{1}(t-\tau)\|\nabla u(t)\|^{2} d \tau-2 \eta_{1}\left(g_{1} \circ \nabla u\right)(t) \\
& \quad-\frac{l_{1}}{2 \eta_{1}}\|\nabla u\|^{2},
\end{aligned}
$$

for any $\eta_{1}>0$. Similarly we have

$$
\begin{aligned}
& 2 \int_{0}^{t} g_{2}(t-\tau) \int_{\Omega} \nabla v(t) \nabla v(\tau) d x d \tau \\
& \quad \geq 2 \int_{0}^{t} g_{2}(t-\tau)\|\nabla v(t)\|^{2} d \tau-2 \eta_{2}\left(g_{2} \circ \nabla v\right)(t) \\
& \quad-\frac{k_{1}}{2 \eta_{2}}\|\nabla v\|^{2}
\end{aligned}
$$

for any $\eta_{2}>0$. Then (84) arrives at

$$
\begin{aligned}
F^{\prime \prime}(t) \geq 2 & \left(\left\|u_{t}\right\|^{2}+\left\|v_{t}\right\|^{2}\right) \\
& -2\left(m_{0}+\alpha\left(\|\nabla u\|^{2}+\|\nabla v\|^{2}\right)^{\gamma}\right)\|\nabla u\|^{2} \\
& +2 \int_{0}^{t} g_{1}(t-\tau)\|\nabla u(t)\|^{2} d \tau \\
& -2 \eta_{1}\left(g_{1} \circ \nabla u\right)(t)-\frac{l_{1}}{2 \eta_{1}}\|\nabla u\|^{2} \\
& -2\left(m_{0}+\alpha\left(\|\nabla u\|^{2}+\|\nabla v\|^{2}\right)^{\gamma}\right)\|\nabla v\|^{2} \\
& +2 \int_{0}^{t} g_{2}(t-\tau)\|\nabla v(t)\|^{2} d \tau \\
& -2 \eta_{2}\left(g_{2} \circ \nabla v\right)(t)-\frac{k_{1}}{2 \eta_{2}}\|\nabla v\|^{2} \\
& +2\left(f_{1}(u, v), u\right)+2\left(f_{2}(u, v), v\right) \\
= & 2\left(\left\|u_{t}\right\|^{2}+\left\|v_{t}\right\|^{2}\right) \\
& -2\left(m_{0}+\alpha\left(\|\nabla u\|^{2}+\|\nabla v\|^{2}\right)^{\gamma}-l_{1}\right)\|\nabla u\|^{2}
\end{aligned}
$$

$$
\begin{aligned}
& -2 \eta_{1}\left(g_{1} \circ \nabla u\right)(t)-\frac{l_{1}}{2 \eta_{1}}\|\nabla u\|^{2} \\
& -2\left(m_{0}+\alpha\left(\|\nabla u\|^{2}+\|\nabla v\|^{2}\right)^{\gamma}-k_{1}\right)\|\nabla v\|^{2} \\
& -2 \eta_{2}\left(g_{2} \circ \nabla v\right)(t)-\frac{k_{1}}{2 \eta_{2}}\|\nabla v\|^{2} \\
& +2\left(f_{1}(u, v), u\right)+2\left(f_{2}(u, v), v\right) \\
= & 2\left(\left\|u_{t}\right\|^{2}+\left\|v_{t}\right\|^{2}\right)-2\left(m_{0}-l_{1}\right)\|\nabla u\|^{2} \\
& -2\left(m_{0}-k_{1}\right)\|\nabla v\|^{2}-2 \eta_{1}\left(g_{1} \circ \nabla u\right)(t) \\
& -\frac{l_{1}}{2 \eta_{1}}\|\nabla u\|^{2}-2 \alpha \beta-2 \eta_{2}\left(g_{2} \circ \nabla v\right)(t) \\
& -\frac{k_{1}}{2 \eta_{2}}\|\nabla v\|^{2}+2(m+1) \int_{\Omega} F(u, v) d x .
\end{aligned}
$$

On the other hand from (83), we have

$$
\begin{aligned}
& \left(F^{\prime}(t)\right)^{2}=4\left(\left(u, u_{t}\right)+\left(v, v_{t}\right)\right)^{2} \\
& \quad+4\left(\int_{0}^{t}\left(\left(u(\tau), u_{\tau}(\tau)\right)+\left(v(\tau), v_{\tau}(\tau)\right)\right) d \tau\right)^{2} \\
& \quad+8\left(\left(u, u_{t}\right)+\left(v, v_{t}\right)\right) \\
& \quad \cdot \int_{0}^{t}\left(\left(u(\tau), u_{\tau}(\tau)\right)+\left(v(\tau), v_{\tau}(\tau)\right)\right) d \tau
\end{aligned}
$$

Using the Schwarz inequality, (88) takes on the form

$$
\begin{aligned}
& \left(\left(u, u_{t}\right)+\left(v, v_{t}\right)\right)^{2} \leq\left(\|u\|^{2}+\|v\|^{2}\right)\left(\left\|u_{t}\right\|^{2}+\left\|v_{t}\right\|^{2}\right) \\
& \left(\int_{0}^{t}\left(u(\tau), u_{\tau}(\tau)\right) d \tau+\int_{0}^{t}\left(v(\tau), v_{\tau}(\tau)\right) d \tau\right)^{2} \\
& \quad \leq \int_{0}^{t}\left(\|u\|^{2}+\|v\|^{2}\right) d \tau \int_{0}^{t}\left(\left\|u_{\tau}\right\|^{2}+\left\|v_{\tau}\right\|^{2}\right) d \tau \\
& 2\left(\left(u, u_{t}\right)+\left(v, v_{t}\right)\right) \\
& \quad \cdot \int_{0}^{t}\left(\left(u(\tau), u_{\tau}(\tau)\right)+\left(v(\tau), v_{\tau}(\tau)\right)\right) d \tau \\
& \quad \leq\left(\left\|u_{\tau}\right\|^{2}+\left\|v_{\tau}\right\|^{2}\right) \int_{0}^{t}\left(\|u\|^{2}+\|v\|^{2}\right) d \tau \\
& \quad+\left(\|u\|^{2}+\|v\|^{2}\right) \int_{0}^{t}\left(\left\|u_{\tau}\right\|^{2}+\left\|v_{\tau}\right\|^{2}\right) d \tau
\end{aligned}
$$

and therefore (88) becomes

$$
\begin{aligned}
& \left(F^{\prime}(t)\right)^{2} \\
& \quad \leq 4\left(\|u\|^{2}+\|v\|^{2}+\int_{0}^{t}\left(\|u\|^{2}+\|v\|^{2}\right) d \tau\right)
\end{aligned}
$$




$$
\begin{gathered}
\cdot\left(\left\|u_{t}\right\|^{2}+\left\|v_{t}\right\|^{2}+\int_{0}^{t}\left(\left\|u_{\tau}\right\|^{2}+\left\|v_{\tau}\right\|^{2}\right) d \tau\right) \\
\leq 4 F(t)\left(\left\|u_{t}\right\|^{2}+\left\|v_{t}\right\|^{2}+\int_{0}^{t}\left(\left\|u_{\tau}\right\|^{2}+\left\|v_{\tau}\right\|^{2}\right) d \tau\right) .
\end{gathered}
$$

Then by (81), (87), and (90), we have

$$
\begin{gathered}
F^{\prime \prime}(t) F(t)-\frac{p+3}{4}\left(F^{\prime}(t)\right)^{2} \geq F(t)\left(F^{\prime \prime}(t)-(p+3)\right. \\
\left.\cdot\left(\left\|u_{t}\right\|^{2}+\left\|v_{t}\right\|^{2}+\int_{0}^{t}\left(\left\|u_{\tau}\right\|^{2}+\left\|v_{\tau}\right\|^{2}\right) d \tau\right)\right) \\
\geq F(t)\left(2\left(\left\|u_{t}\right\|^{2}+\left\|v_{t}\right\|^{2}\right)-2\left(m_{0}-l_{1}\right)\|\nabla u\|^{2}\right. \\
\left.-2\left(m_{0}-k_{1}\right)\|\nabla v\|^{2}\right)-F(t)\left(2 \eta_{1}\left(g_{1} \circ \nabla u\right)(t)\right. \\
\left.+\frac{l_{1}}{2 \eta_{1}}\|\nabla u\|^{2}+2 \alpha \beta\right)-F(t)\left(2 \eta_{2}\left(g_{2} \circ \nabla v\right)(t)\right. \\
\left.+\frac{k_{1}}{2 \eta_{2}}\|\nabla v\|^{2}-2(m+1) \int_{\Omega} F(u, v) d x\right)-F(t)(m \\
+3)\left(\left\|u_{t}\right\|^{2}+\left\|v_{t}\right\|^{2}+\int_{0}^{t}\left(\left\|u_{\tau}\right\|^{2}+\left\|v_{\tau}\right\|^{2}\right) d \tau\right) .
\end{gathered}
$$

Now we define

$$
\begin{aligned}
& \xi(t):=2\left(\left\|u_{t}\right\|^{2}+\left\|v_{t}\right\|^{2}\right)-2\left(m_{0}-l_{1}\right)\|\nabla u\|^{2} \\
&- 2\left(m_{0}-k_{1}\right)\|\nabla v\|^{2}-2 \eta_{1}\left(g_{1} \circ \nabla u\right)(t) \\
&- \frac{l_{1}}{2 \eta_{1}}\|\nabla u\|^{2}-2 \alpha \beta-2 \eta_{2}\left(g_{2} \circ \nabla v\right)(t) \\
&- \frac{k_{1}}{2 \eta_{2}}\|\nabla v\|^{2}+2(m+1) \int_{\Omega} F(u, v) d x-(m+3) \\
& \cdot\left(\left\|u_{t}\right\|^{2}+\left\|v_{t}\right\|^{2}+\int_{0}^{t}\left(\left\|u_{\tau}\right\|^{2}+\left\|v_{\tau}\right\|^{2}\right) d \tau\right) .
\end{aligned}
$$

From the definition of $E(t)$, (92) becomes

$$
\begin{aligned}
\xi(t):= & (m-1)\left(m_{0}-l_{1}\right)\|\nabla u\|^{2} \\
& +(m-1)\left(m_{0}-k_{1}\right)\|\nabla v\|^{2} \\
& +\left(m+1-2 \eta_{1}\right)\left(g_{1} \circ \nabla u\right)(t) \\
& +\left(m+1-2 \eta_{2}\right)\left(g_{2} \circ \nabla v\right)(t)-\frac{k_{1}}{2 \eta_{2}}\|\nabla v\|^{2} \\
& -\frac{l_{1}}{2 \eta_{1}}\|\nabla u\|^{2}+\left(\frac{m+1}{r+1}-2\right) \alpha \beta \\
& -(m+3) \int_{0}^{t}\left(\left\|u_{\tau}\right\|^{2}+\left\|v_{\tau}\right\|^{2}\right) d \tau \\
& -2(m+1) E(t) .
\end{aligned}
$$

Then from Lemma 2 with $p=q=1$, (93) arrives at

$$
\begin{aligned}
& \xi(t):=(m-1)\left(m_{0}-l_{1}\right)\|\nabla u\|^{2}+(m-1)\left(m_{0}-k_{1}\right) \\
& \cdot\|\nabla v\|^{2}+\left(m+1-2 \eta_{1}\right)\left(g_{1} \circ \nabla u\right)(t)-\frac{l_{1}}{2 \eta_{1}}\|\nabla u\|^{2} \\
& +\left(m+1-2 \eta_{2}\right)\left(g_{2} \circ \nabla v\right)(t)-\frac{k_{1}}{2 \eta_{2}}\|\nabla v\|^{2} \\
& +\left(\frac{m+1}{r+1}-2\right) \alpha \beta-2(m+1) E(0)+(m+1) \\
& \cdot \int_{0}^{t}\left(g_{1}(s)\|\nabla u(s)\|^{2}+g_{2}(s)\|\nabla v(s)\|^{2}\right) d s \\
& \quad-(m+1) \int_{0}^{t}\left(\left(g_{1}^{\prime} \circ \nabla u\right)(s)+\left(g_{2}^{\prime} \circ \nabla v\right)(s)\right) d s .
\end{aligned}
$$

From assumption (A4) on $g_{1}$ and $g_{2}$ we can derive

$$
\begin{aligned}
\xi(t) \geq & (m-1)\left(m_{0}-l_{1}\right)\|\nabla u\|^{2} \\
& +(m-1)\left(m_{0}-k_{1}\right)\|\nabla v\|^{2} \\
& +\left(m+1-2 \eta_{1}\right)\left(g_{1} \circ \nabla u\right)(t)-\frac{l_{1}}{2 \eta_{1}}\|\nabla u\|^{2} \\
& +\left(m+1-2 \eta_{2}\right)\left(g_{2} \circ \nabla v\right)(t)-\frac{k_{1}}{2 \eta_{2}}\|\nabla v\|^{2} \\
& +\left(\frac{m+1}{r+1}-2\right) \alpha \beta-2(m+1) E(0) \\
= & \left.(m-1) m_{0}-\left((m-1)+\frac{1}{2 \eta_{1}}\right) l_{1}\right)\|\nabla u\|^{2} \\
& +\left((m-1) m_{0}-\left((m-1)+\frac{1}{2 \eta_{2}}\right) k_{1}\right)\|\nabla v\|^{2} \\
& +\left(m+1-2 \eta_{1}\right)\left(g_{1} \circ \nabla u\right)(t) \\
& +\left(m+1-2 \eta_{2}\right)\left(g_{2} \circ \nabla v\right)(t) \\
& +\left(\frac{m+1}{r+1}-2\right) \alpha \beta-2(m+1) E(0) \\
= & \left((m-1) m_{0}-\left((m-1)+\frac{1}{2 \eta_{1}}\right) l_{1}\right)\|\nabla u\|^{2} \\
& +\zeta(m-1)\left(m_{0}-m_{1}\right)\left(\|\nabla u\|^{2}+\|\nabla v\|^{2}\right) \\
& +\left((m-1) m_{0}-\left((m-1)+\frac{1}{2 \eta_{2}}\right) k_{1}\right)\|\nabla v\|^{2} \\
& +\left(m+1-2 \eta_{1}\right)\left(g_{1} \circ \nabla u\right)(t) \\
& +\left(m+1-2 \eta_{2}\right)\left(g_{2} \circ \nabla v\right)(t) \\
& (m-1)\left(m_{0}-m_{1}\right)\left(\|\nabla u\|^{2}+\|\nabla v\|^{2}\right) \\
& +\zeta d+\left(\frac{m+1}{r+1}-2\right) \alpha \beta \\
& (m)
\end{aligned}
$$




$$
\begin{aligned}
& +2(m+1) \zeta d-2(m+1) E(0) \\
= & \xi_{1}+\xi_{2}+\xi_{3},
\end{aligned}
$$

where

$$
\begin{aligned}
\xi_{1}= & \left((m-1) m_{0}-\left((m-1)+\frac{1}{2 \eta_{1}}\right) l_{1}\right)\|\nabla u\|^{2} \\
& +\left((m-1) m_{0}-\left((m-1)+\frac{1}{2 \eta_{2}}\right) k_{1}\right)\|\nabla v\|^{2} \\
& +\left(m+1-2 \eta_{1}\right)\left(g_{1} \circ \nabla u\right)(t) \\
& +\left(m+1-2 \eta_{2}\right)\left(g_{2} \circ \nabla v\right)(t) \\
& -\zeta(m-1)\left(m_{0}-m_{1}\right)\left(\|\nabla u\|^{2}+\|\nabla v\|^{2}\right), \\
\xi_{2}= & \zeta(m-1)\left(m_{0}-m_{1}\right)\left(\|\nabla u\|^{2}+\|\nabla v\|^{2}\right) \\
& -2(m+1) \zeta d, \\
\xi_{3}= & \left(\frac{m+1}{r+1}-2\right) \alpha \beta+2(m+1) \zeta d \\
& -2(m+1) E(0) .
\end{aligned}
$$

We next estimate the terms $\xi_{1}, \xi_{2}$, and $\xi_{3}$ one by one as follows. For the term $\xi_{1}$ from

$$
m_{1}=\max \left\{l_{1}, k_{1}\right\}
$$

we have

$$
\begin{aligned}
\xi_{1} & =\left((m-1) m_{0}-\left((m-1)+\frac{1}{2 \eta_{1}}\right) l_{1}\right)\|\nabla u\|^{2} \\
& +\left((m-1) m_{0}-\left((m-1)+\frac{1}{2 \eta_{2}}\right) k_{1}\right)\|\nabla v\|^{2} \\
& +\left(m+1-2 \eta_{1}\right)\left(g_{1} \circ \nabla u\right)(t)+\left(m+1-2 \eta_{2}\right)\left(g_{2}\right. \\
& \circ \nabla v)(t)-\zeta(m-1)\left(m_{0}-m_{1}\right)\left(\|\nabla u\|^{2}+\|\nabla v\|^{2}\right) \\
& \geq\left((m-1) m_{0}-\left((m-1)+\frac{1}{2 \eta_{1}}\right) m_{1}\right)\|\nabla u\|^{2} \\
& +\left((m-1) m_{0}-\left((m-1)+\frac{1}{2 \eta_{2}}\right) m_{1}\right)\|\nabla v\|^{2} \\
& +\left(m+1-2 \eta_{1}\right)\left(g_{1} \circ \nabla u\right)(t)+\left(m+1-2 \eta_{2}\right)\left(g_{2}\right. \\
& \circ \nabla v)(t)-\zeta(m-1)\left(m_{0}-m_{1}\right)\left(\|\nabla u\|^{2}+\|\nabla v\|^{2}\right) \\
& =\left((m-1)(1-\zeta) m_{0}\right. \\
& \left.-\left((m-1)(1-\zeta)+\frac{1}{2 \eta_{1}}\right) m_{1}\right)\|\nabla u\|^{2} \\
& ((m)-(m)
\end{aligned}
$$

$$
\begin{aligned}
& +\left((m-1)(1-\zeta) m_{0}\right. \\
& \left.-\left((m-1)(1-\zeta)+\frac{1}{2 \eta_{2}}\right) m_{1}\right)\|\nabla v\|^{2}+(m+1 \\
& \left.-2 \eta_{1}\right)\left(g_{1} \circ \nabla u\right)(t)+\left(m+1-2 \eta_{2}\right)\left(g_{2} \circ \nabla v\right)(t) .
\end{aligned}
$$

Here by taking $2 \eta_{1}=m+1$ and $2 \eta_{2}=m+1$ and by (80) we have

$$
\xi_{1}>0 \text {. }
$$

From Lemma 16 we can derive

$$
\xi_{2}>0 \text {. }
$$

With the fact $E(0)<\zeta d$ we have

$$
\xi_{3}>0 .
$$

So from (95) we have $\xi(t)>\sigma_{1}>0$. Therefore we can derive

$$
F^{\prime \prime}(t) F(t)-\frac{p+3}{4} F^{\prime}(t)^{2} \geq \rho \sigma_{1}>0, \quad t \in\left[0, T_{0}\right] .
$$

Setting $y(t)=F(t)^{-(p-1) / 4}$, this inequality becomes

$$
y^{\prime \prime}(t) \leq-\frac{p-1}{4} \sigma_{1} \rho y(t)^{(p+7) /(p-1)}, \quad t \in\left[0, T_{0}\right] .
$$

This proves that $y(t)$ reaches 0 in finite time, say $t \rightarrow T_{*}$. Since $T_{*}$ is independent of the initial choice of $T_{0}$, we may assume that $T_{*}<T_{0}$. This tells us that

$$
\lim _{t \rightarrow T_{*}} F(t)=+\infty
$$

which completes the proof.

\section{A Finite Time Blow-Up When $E(0)>0$}

We first present the following lemmas in order to prove Theorem 18.

Lemma 15. Let condition (A4) hold and the nonlinear viscoelastic terms $g_{1}$ and $g_{2}$ satisfy

$$
\begin{gathered}
\int_{0}^{t} w(s) \int_{0}^{s} e^{(s-\tau) / 2} g_{1}(s-\tau) w(\tau) d \tau d s \geq 0, \\
\forall w \in C^{1}([0, \infty)), \forall t>0 \\
\int_{0}^{t} w(s) \int_{0}^{s} e^{(s-\tau) / 2} g_{2}(s-\tau) w(\tau) d \tau d s \geq 0, \\
\forall w \in C^{1}([0, \infty)), \forall t>0
\end{gathered}
$$

If $H(t)$ is a twice continuously differentiable function and satisfies the inequality

$$
\begin{aligned}
& H^{\prime \prime}(t)+H^{\prime}(t) \\
& \quad>\int_{0}^{t} g_{1}(t-\tau) \int_{\Omega} \nabla u(x, \tau) \nabla u(x, t) d x d \tau \\
& \quad+\int_{0}^{t} g_{2}(t-\tau) \int_{\Omega} \nabla v(x, \tau) \nabla v(x, t) d x d \tau
\end{aligned}
$$


and the initial condition

$$
\begin{aligned}
& H(0)>0, \\
& H^{\prime}(0)>0,
\end{aligned}
$$

for every $t \in\left[0, T_{0}\right)$, where $(u(t), v(t))$ is the corresponding solutions of problem (1)-(5) with $\left(u_{0}, v_{0}\right)$ and $\left(u_{1}, v_{1}\right)$, then the function $H(t)$ is strictly increasing on $\left[0, T_{0}\right)$.

Proof. Consider the following auxiliary ordinary differential equation:

$$
\begin{aligned}
& h^{\prime \prime}(t)+h^{\prime}(t) \\
&= \int_{0}^{t} g_{1}(t-\tau) \int_{\Omega} \nabla u(x, \tau) \nabla u(x, t) d x d \tau \\
& \quad+\int_{0}^{t} g_{2}(t-\tau) \int_{\Omega} \nabla v(x, \tau) \nabla v(x, t) d x d \tau,
\end{aligned}
$$

with the initial condition

$$
\begin{aligned}
& h(0)=H(0), \\
& h^{\prime}(0)=0,
\end{aligned}
$$

for every $t \in\left[0, T_{0}\right)$.

Clearly we can find the following function:

$$
\begin{gathered}
h(t)=h(0)+\int_{0}^{t} \frac{e^{-\xi}-e^{-t}}{e^{-\xi}} \int_{0}^{\xi} g_{1}(\xi-\tau) \\
\cdot \int_{\Omega} \nabla u(x, \tau) \nabla u(x, \xi) d x d \tau d \xi \\
+\int_{0}^{t} \frac{e^{-\xi}-e^{-t}}{e^{-\xi}} \int_{0}^{\xi} g_{2}(\xi-\tau) \\
\cdot \int_{\Omega} \nabla v(x, \tau) \nabla v(x, \xi) d x d \tau d \xi
\end{gathered}
$$

as a solution of the ODE (109) and (110) for every $t \in\left[0, T_{0}\right)$.

Now in order to show that

$$
H^{\prime}(t)>0, \quad t \geq 0,
$$

we need to prove that

$$
H^{\prime}(t)>h^{\prime}(t) \geq 0, \quad t \geq 0 .
$$

From (105) a direct computation on (111) yields

$$
\begin{aligned}
& h^{\prime}(t)=\int_{0}^{t} e^{\xi-t} \int_{0}^{\xi} g_{1}(\xi-\tau) \\
& \cdot \int_{\Omega} \nabla u(x, \tau) \nabla u(x, \xi) d x d \tau d \xi \\
& +\int_{0}^{t} e^{\xi-t} \int_{0}^{\xi} g_{2}(\xi-\tau) \\
& \cdot \int_{\Omega} \nabla v(x, \tau) \nabla v(x, \xi) d x d \tau d \xi
\end{aligned}
$$

$$
=e^{-t} \int_{\Omega} e^{\xi} \int_{0}^{t} \int_{0}^{\xi} g_{1}(\xi-\tau)
$$

$\cdot \nabla u(x, \tau) \nabla u(x, \xi) d \tau d \xi d x$

$+e^{-t} \int_{\Omega} e^{\xi} \int_{0}^{t} \int_{0}^{\xi} g_{2}(\xi-\tau)$

$\cdot \nabla v(x, \tau) \nabla v(x, \xi) d \tau d \xi d x$

$=e^{-t} \int_{\Omega} \int_{0}^{t} \int_{0}^{\xi} e^{(\xi-\tau) / 2} g_{1}(\xi-\tau) e^{\tau / 2}$

$\cdot \nabla u(x, \tau) e^{\xi / 2} \nabla u(x, \xi) d \tau d \xi d x$

$+e^{-t} \int_{\Omega} \int_{0}^{t} \int_{0}^{\xi} e^{(\xi-\tau) / 2} g_{2}(\xi-\tau) e^{\tau / 2}$

$\cdot \nabla v(x, \tau) e^{\xi / 2} \nabla v(x, \xi) d \tau d \xi d x$

$=e^{-t} \int_{\Omega} \int_{0}^{t}\left(e^{\xi / 2} \nabla u(x, \xi)\right) \int_{0}^{\xi}\left(e^{(\xi-\tau) / 2} g_{1}(\xi-\tau)\right)$

$\cdot\left(e^{\tau / 2} \nabla u(x, \tau)\right) d \tau d \xi d x$

$+e^{-t} \int_{\Omega} \int_{0}^{t}\left(e^{\xi / 2} \nabla v(x, \xi)\right) \int_{0}^{\xi}\left(e^{(\xi-\tau) / 2} g_{2}(\xi-\tau)\right)$

$\cdot\left(e^{\tau / 2} \nabla v(x, \tau)\right) d \tau d \xi d x \geq 0$,

for every $t \in\left[0, T_{0}\right)$, which says that

$$
h(t) \geq h(0)=H(0) .
$$

Moreover from (108) and (114) it implies that

$$
H^{\prime}(0)>0=h^{\prime}(0) \text {. }
$$

Suppose by contradiction that the first inequality of (113) is invalid; then there exists $t_{1} \in\left[0, T_{0}\right)$ such that

$$
H^{\prime}\left(t_{1}\right) \leq h^{\prime}\left(t_{1}\right) \text {. }
$$

From the continuity of the solution in time, there exists $t_{0} \epsilon$ $\left[0, T_{0}\right)$ such that

$$
H^{\prime}\left(t_{0}\right)=h^{\prime}\left(t_{0}\right) \text {. }
$$

On the other hand we have the following ordinary differential inequality

$$
\begin{aligned}
\left(H^{\prime \prime}(t)-h^{\prime \prime}(t)\right)+( & \left.H^{\prime}(t)-h^{\prime}(t)\right)>0, \\
H(0)-h(0) & =0, \\
H^{\prime}(0)-h^{\prime}(0) & >0,
\end{aligned}
$$

for every $t \in\left[0, T_{0}\right)$. This ordinary differential inequality can be solved as

$$
H^{\prime}\left(t_{0}\right)-h^{\prime}\left(t_{0}\right)>e^{-t_{0}}\left(H^{\prime}(0)-h^{\prime}(0)\right)>0,
$$

which contradicts (118). Thus we prove the first inequality of (113), which together with (114) states (113). So we complete this proof. 
Lemma 16. Let $\left(u_{0}, v_{0}\right) \in H_{0}^{1}(\Omega) \times H_{0}^{1}(\Omega),\left(u_{1}, v_{1}\right) \in L^{2}(\Omega) \times$ $L^{2}(\Omega), p=q=1$, and $(u, v)$ be the solution of problem (1)-(5) with the initial data $\left(u_{0}, v_{0}\right)$ and $\left(u_{1}, v_{1}\right)$. Assume the initial data satisfy

$$
\left(u_{0}, u_{1}\right)+\left(v_{0}, v_{1}\right) \geq 0
$$

then the map

$$
\left\{t \longmapsto\|u(t)\|^{2}+\|v(t)\|^{2}\right\}
$$

is strictly increasing as long as $(u, v) \in V$.

Proof. Let

$$
H(t)=\|u\|^{2}+\|v\|^{2}
$$

then we have

$$
\begin{aligned}
H^{\prime}(t)= & 2\left(u, u_{t}\right)+2\left(v, v_{t}\right), \\
H^{\prime \prime}(t)= & 2\left(\left\|u_{t}\right\|^{2}+\left\|v_{t}\right\|^{2}\right)+2\left(u, u_{t t}\right)+2\left(v, v_{t t}\right) \\
= & 2\left(\left\|u_{t}\right\|^{2}+\left\|v_{t}\right\|^{2}\right)-2\left(u, u_{t}\right)-2\left(v, v_{t}\right) \\
& +2 \int_{0}^{t} g_{1}(t-\tau) \int_{\Omega} \nabla u(t) \nabla u(\tau) d x d \tau \\
& +2 \int_{0}^{t} g_{2}(t-\tau) \int_{\Omega} \nabla v(t) \nabla v(\tau) d x d \tau \\
& -2\left(m_{0}+\alpha\left(\|\nabla u\|^{2}+\|\nabla v\|^{2}\right)^{\gamma}\right)\|\nabla u\|^{2} \\
& -2\left(m_{0}+\alpha\left(\|\nabla u\|^{2}+\|\nabla v\|^{2}\right)^{\gamma}\right)\|\nabla v\|^{2} \\
& +2\left(f_{1}(u, v), u\right)+2\left(f_{2}(u, v), v\right) \\
= & 2\left(\left\|u_{t}\right\|^{2}+\left\|v_{t}\right\|^{2}\right)-2\left(u, u_{t}\right)-2\left(v, v_{t}\right) \\
& -2 I(u, v) \\
& +2 \int_{0}^{t} g_{1}(t-\tau) \int_{\Omega} \nabla u(t) \nabla u(\tau) d x d \tau \\
& +2 \int_{0}^{t} g_{2}(t-\tau) \int_{\Omega} \nabla v(t) \nabla v(\tau) d x d \tau .
\end{aligned}
$$

Adding (124) and (125) we have

$$
\begin{aligned}
H^{\prime \prime}(t) & +H^{\prime}(t) \\
= & 2\left(\left\|u_{t}\right\|^{2}+\left\|v_{t}\right\|^{2}\right)-2 I(u, v) \\
& +2 \int_{0}^{t} g_{1}(t-\tau) \int_{\Omega} \nabla u(t) \nabla u(\tau) d x d \tau \\
& +2 \int_{0}^{t} g_{2}(t-\tau) \int_{\Omega} \nabla v(t) \nabla v(\tau) d x d \tau,
\end{aligned}
$$

which, from the fact that $(u, v) \in V$, implies that

$$
\begin{aligned}
& H^{\prime \prime}(t)+H^{\prime}(t) \\
& \geq 2 \int_{0}^{t} g_{1}(t-\tau) \int_{\Omega} \nabla u(t) \nabla u(\tau) d x d \tau \\
& \quad+2 \int_{0}^{t} g_{2}(t-\tau) \int_{\Omega} \nabla v(t) \nabla v(\tau) d x d \tau .
\end{aligned}
$$

Therefore, applying Lemma 15 with the fact that

$$
H^{\prime}(0)=2 \int_{\Omega} u_{0} u_{1} d x+2 \int_{\Omega} v_{0} v_{1} d x \geq 0,
$$

we can obtain that the map

$$
\left\{t \longmapsto\|u(t)\|^{2}+\|v(t)\|^{2}\right\}
$$

is strictly increasing.

In the following, we show the invariance of the unstable set under the flow of the problem (1)-(5).

Lemma 17. Let $\left(u_{0}, v_{0}\right) \in H_{0}^{1}(\Omega) \times H_{0}^{1}(\Omega),\left(u_{1}, v_{1}\right) \in L^{2}(\Omega) \times$ $L^{2}(\Omega), p=q=1$, and $(u, v)$ be the solution of problem (1)-(5) with the initial data $\left(u_{0}, v_{0}\right)$ and $\left(u_{1}, v_{1}\right)$. Assume that the nonlinear viscoelastic terms $g_{1}$ and $g_{2}$ satisfy

$$
m_{1}<\frac{(m-1) m_{0}}{(m-1)+1 /(m+1)},
$$

and the initial data satisfy (121) and

$$
\left\|u_{0}\right\|^{2}+\left\|v_{0}\right\|^{2}>\frac{2(m+1)}{A C} E(0),
$$

where

$$
\begin{aligned}
& A=(m-1) m_{0}-\left((m-1)+\frac{1}{m+1}\right) m_{1}, \\
& C=\min \left\{C_{1}, C_{2}\right\} .
\end{aligned}
$$

$C_{1}$ is the coefficient of Poincare inequality $\|\nabla u\|^{2} \geq C_{1}\|u\|^{2}$ and $C_{2}$ is the coefficient of Poincaré inequality $\|\nabla v\|^{2} \geq C_{2}\|v\|^{2}$. Then all solutions of problem (1)-(5) with $E(0)>0$ belong to $V$, provided $I\left(u_{0}, v_{0}\right)<0$.

Proof. We prove $(u(t), v(t)) \in V$. If it is false, let $t_{0} \in(0, T)$ be the first time such that $I(u(t), v(t))=0$; that is, $I(u(t), v(t))<$ 0 , for $t \in\left[0, t_{0}\right)$ and $I\left(u\left(t_{0}\right), v\left(t_{0}\right)\right)=0$. Now let $H(t)$ be defined by (123) above. Hence from Lemma 16, we get that $H(t)$ and $H^{\prime}(t)$ are strictly increasing on the interval $\left(0, t_{0}\right)$. And then by (131), we have

$$
\begin{aligned}
& H(t)>\left\|v_{0}\right\|^{2}+\left\|u_{0}\right\|^{2}>\frac{2(m+1)}{A C} E(0), \\
& \forall t \in\left[0, t_{0}\right) .
\end{aligned}
$$

Moreover, from the continuity of $u(t)$ in $t$, we obtain

$$
H\left(t_{0}\right)>\frac{2(m+1)}{A C} E(0) .
$$


On the other hand, by (37) and (39) we can obtain

$$
\begin{aligned}
E(0) & \geq E\left(t_{0}\right)=\frac{1}{2}\left\|u_{t}\left(t_{0}\right)\right\|^{2}+\frac{1}{2}\left\|v_{t}\left(t_{0}\right)\right\|^{2} \\
+ & \frac{\alpha \beta\left(t_{0}\right)}{2(\gamma+1)}+\frac{1}{2}\left(m_{0}-l_{1}\right)\left\|\nabla u\left(t_{0}\right)\right\|^{2} \\
+ & \frac{1}{2}\left(m_{0}-k_{1}\right)\left\|\nabla v\left(t_{0}\right)\right\|^{2}+\frac{1}{2}\left(g_{1} \circ \nabla u\right)\left(t_{0}\right) \\
+ & \frac{1}{2}\left(g_{2} \circ \nabla v\right)\left(t_{0}\right)-\int_{\Omega} F\left(u\left(t_{0}\right), v\left(t_{0}\right)\right) d x \\
= & \frac{1}{2}\left\|u_{t}\left(t_{0}\right)\right\|^{2}+\frac{1}{2}\left\|v_{t}\left(t_{0}\right)\right\|^{2} \\
+ & \left(\frac{\alpha}{2(\gamma+1)}-\frac{\alpha}{m+1}\right) \beta\left(t_{0}\right)+\left(\frac{1}{2}-\frac{1}{m+1}\right) \\
& \cdot\left(\left(m_{0}-l_{1}\right)\left\|\nabla u\left(t_{0}\right)\right\|^{2}+\left(m_{0}-k_{1}\right)\left\|\nabla v\left(t_{0}\right)\right\|^{2}\right) \\
+ & \left(\frac{1}{2}-\frac{1}{m+1}\right)\left(g_{1} \circ \nabla u\right)\left(\left(t_{0}\right)\right)+\left(\frac{1}{2}-\frac{1}{m+1}\right) \\
& \cdot\left(g_{2} \circ \nabla v\right)\left(\left(t_{0}\right)\right)+\frac{1}{m+1} I\left(u\left(t_{0}\right), v\left(t_{0}\right)\right) .
\end{aligned}
$$

Note that $I\left(u\left(t_{0}\right), v\left(t_{0}\right)\right)=0$; hence we have

$$
\begin{aligned}
E(0) & \geq \frac{1}{2}\left\|u_{t}\left(t_{0}\right)\right\|^{2}+\frac{1}{2}\left\|v_{t}\left(t_{0}\right)\right\|^{2} \\
+ & \left(\frac{\alpha}{2(\gamma+1)}-\frac{\alpha}{m+1}\right) \beta\left(t_{0}\right)+\left(\frac{1}{2}-\frac{1}{m+1}\right) \\
\cdot & \left(\left(m_{0}-l_{1}\right)\left\|\nabla u\left(t_{0}\right)\right\|^{2}+\left(m_{0}-k_{1}\right)\left\|\nabla v\left(t_{0}\right)\right\|^{2}\right) \\
+ & \left(\frac{1}{2}-\frac{1}{m+1}\right)\left(g_{1} \circ \nabla u\right)\left(\left(t_{0}\right)\right)+\left(\frac{1}{2}-\frac{1}{m+1}\right) \\
\cdot & \left(g_{2} \circ \nabla v\right)\left(\left(t_{0}\right)\right) \geq\left(\frac{1}{2}-\frac{1}{m+1}\right) \\
\cdot & \left(\left(m_{0}-l_{1}\right)\left\|\nabla u\left(t_{0}\right)\right\|^{2}+\left(m_{0}-k_{1}\right)\left\|\nabla v\left(t_{0}\right)\right\|^{2}\right) \\
\geq & \left(\frac{1}{2}-\frac{1}{m+1}\right)\left(m_{0}-m_{1}\right) \\
\cdot & \left(\left\|\nabla u\left(t_{0}\right)\right\|^{2}+\left\|\nabla v\left(t_{0}\right)\right\|^{2}\right) .
\end{aligned}
$$

Using the Poincaré inequality

$$
\begin{gathered}
\|\nabla u\|^{2} \geq C_{1}\|u\|^{2}, \\
\|\nabla v\|^{2} \geq C_{2}\|v\|^{2},
\end{gathered}
$$

we have

$$
\begin{aligned}
\|\nabla u\|^{2}+\|\nabla v\|^{2} & \geq C_{1}\|u\|^{2}+C_{2}\|v\|^{2} \\
& \geq C\left(\|u\|^{2}+\|v\|^{2}\right) .
\end{aligned}
$$

By (138), we deduce (136) to

$$
\begin{aligned}
E(0) & \geq\left(\frac{1}{2}-\frac{1}{m+1}\right)\left(m_{0}-m_{1}\right) \\
\cdot & \left(\left\|\nabla u\left(t_{0}\right)\right\|^{2}+\left\|\nabla v\left(t_{0}\right)\right\|^{2}\right) \geq\left(\frac{1}{2}-\frac{1}{m+1}\right) \\
\cdot & \left(m_{0}-m_{1}\right) C\left(\left\|u\left(t_{0}\right)\right\|^{2}+\left\|v\left(t_{0}\right)\right\|^{2}\right) \\
\geq & \left(\frac{1}{2}-\frac{1}{m+1}\right)\left(m_{0}-m_{1}\right) \\
\cdot & C\left(\left\|u\left(t_{0}\right)\right\|^{2}+\left\|v\left(t_{0}\right)\right\|^{2}\right)-\frac{m_{1}}{2(m+1)^{2}} \\
\cdot & C\left(\left\|u\left(t_{0}\right)\right\|^{2}+\left\|v\left(t_{0}\right)\right\|^{2}\right) \\
= & \frac{(m-1) m_{0}-(m-1) m_{1}-m_{1} /(m+1)}{2(m+1)} \\
\cdot & C\left(\left\|u\left(t_{0}\right)\right\|^{2}+\left\|v\left(t_{0}\right)\right\|^{2}\right) \\
= & \frac{A C}{2(m+1)}\left(\left\|u\left(t_{0}\right)\right\|^{2}+\left\|v\left(t_{0}\right)\right\|^{2}\right)
\end{aligned}
$$

which means

$$
H\left(t_{0}\right)=\left\|u\left(t_{0}\right)\right\|^{2}+\left\|v\left(t_{0}\right)\right\|^{2} \leq \frac{2(m+1)}{A C} E(0) .
$$

It is obvious that (140) contradicts (131).

Theorem 18 (finite time blow-up under the case of $E(0)>0$ and $p=q=1)$. Let $\left(u_{0}, v_{0}\right) \in H_{0}^{1}(\Omega) \times H_{0}^{1}(\Omega),\left(u_{1}, v_{1}\right) \in$ $L^{2}(\Omega) \times L^{2}(\Omega)$, and (A1)-(A4) hold. Assume that the nonlinear viscoelastic terms $g_{1}$ and $g_{2}$ satisfy (105), (106), and (130) and the initial data satisfy (121), (131), and $\left(u_{0}, v_{0}\right) \in V$. Then the solution of problem (1)-(5) with $p=q=1$ and $E(0)>0$ blows up in finite time.

Proof. Recalling the auxiliary function $F(t)$ defined as (81) and the proof of Theorem 14, we have

$\xi(t)$

$$
\begin{aligned}
& \geq(m-1)\left(m_{0}-l_{1}\right)\|\nabla u\|^{2} \\
& \quad+(m-1)\left(m_{0}-k_{1}\right)\|\nabla v\|^{2} \\
& \quad+\left(m+1-2 \eta_{1}\right)\left(g_{1} \circ \nabla u\right)(t)-\frac{l_{1}}{2 \eta_{1}}\|\nabla u\|^{2} \\
& \quad+\left(m+1-2 \eta_{2}\right)\left(g_{2} \circ \nabla v\right)(t)-\frac{k_{1}}{2 \eta_{2}}\|\nabla v\|^{2} \\
& \quad+\left(\frac{m+1}{r+1}-2\right) \alpha \beta-2(m+1) E(0) \\
& =\left((m-1) m_{0}-\left((m-1)+\frac{1}{2 \eta_{1}}\right) l_{1}\right)\|\nabla u\|^{2} \\
& \quad+\left((m-1) m_{0}-\left((m-1)+\frac{1}{2 \eta_{2}}\right) k_{1}\right)\|\nabla v\|^{2}
\end{aligned}
$$




$$
\begin{aligned}
& +\left(m+1-2 \eta_{1}\right)\left(g_{1} \circ \nabla u\right)(t) \\
& +\left(m+1-2 \eta_{2}\right)\left(g_{2} \circ \nabla v\right)(t) \\
& +\left(\frac{m+1}{r+1}-2\right) \alpha \beta-2(m+1) E(0) \\
& \geq\left((m-1) m_{0}-\left((m-1)+\frac{1}{2 \eta_{1}}\right) m_{1}\right)\|\nabla u\|^{2} \\
& +\left((m-1) m_{0}-\left((m-1)+\frac{1}{2 \eta_{2}}\right) m_{1}\right)\|\nabla v\|^{2} \\
& +\left(m+1-2 \eta_{1}\right)\left(g_{1} \circ \nabla u\right)(t) \\
& +\left(m+1-2 \eta_{2}\right)\left(g_{2} \circ \nabla v\right)(t) \\
& +\left(\frac{m+1}{r+1}-2\right) \alpha \beta-2(m+1) E(0) .
\end{aligned}
$$

Since (141) holds for any $0<\eta_{1}, \eta_{2} \leq(m+1) / 2$, we can choose $\eta_{1}=\eta_{2}=(m+1) / 2$; then (141) becomes

$$
\begin{aligned}
\xi(t) & \geq\left((m-1) m_{0}-\left((m-1)+\frac{1}{m+1}\right) m_{1}\right) \\
\cdot & \left(\|\nabla u\|^{2}+\|\nabla v\|^{2}\right)+\left(\frac{m+1}{r+1}-2\right) \alpha \beta-2(m+1) \\
\cdot & E(0) \geq\left((m-1) m_{0}-\left((m-1)+\frac{1}{m+1}\right) m_{1}\right) \\
\cdot & \left(\|\nabla u\|^{2}+\|\nabla v\|^{2}\right)-2(m+1) E(0) .
\end{aligned}
$$

Then from Lemma 17 and Poincaré inequality, we conclude that

$$
\begin{aligned}
\xi(t) & \geq\left((m-1) m_{0}-\left((m-1)+\frac{1}{m+1}\right) m_{1}\right) \\
\cdot & \left(\|\nabla u\|^{2}+\|\nabla v\|^{2}\right)+\left(\frac{m+1}{r+1}-2\right) \alpha \beta-2(m+1) \\
\cdot & E(0) \geq\left((m-1) m_{0}-\left((m-1)+\frac{1}{m+1}\right) m_{1}\right) \\
\cdot & \left(\|\nabla u\|^{2}+\|\nabla v\|^{2}\right)-2(m+1) E(0) \\
\geq & \left((m-1) m_{0}-\left((m-1)+\frac{1}{m+1}\right) m_{1}\right) \\
\cdot C & \left(\|u\|^{2}+\|v\|^{2}\right)-2(m+1) E(0),
\end{aligned}
$$

which means $\xi(t)>\sigma>0$. Similar to the proof of Theorem 14, by the concavity argument, we conclude the result.

\section{Conflicts of Interest}

The authors declare that they have no conflicts of interest.

\section{Acknowledgments}

This work was supported by the National Natural Science Foundation of China (11071033 and 11101102), the PhD StartUp Fund of Liaoning Province of China (nos. 20141137 and 20141139), the Natural Science Foundation of Liaoning Province of China (no. 20170540004), and the Liaoning BaiQianWan Talents Program (no. 2013921055).

\section{References}

[1] G. O. Carrier, B. L. Pegram, and O. Carrier Jr., "Antagonistic effects of reserpine on d-tubocurarine action on motor function of rabbits," European Journal of Pharmacology, vol. 6, no. 2, pp. 125-128, 1969.

[2] S. I. Pohozaev and A. Tesei, "Nonexistence of local solutions to semilinear partial differential inequalities," Annales de l'Institut Henri Poincare. Analyse Non Lineaire, vol. 21, no. 4, pp. 487-502, 2004.

[3] R. Torrejon and J. M. Yong, "On a quasilinear wave equation with memory," Nonlinear Analysis. Theory, Methods \& Applications, vol. 16, no. 1, pp. 61-78, 1991.

[4] J. E. Munoz Rivera, "Global solution on a quasilinear wave equation with memory," Bollettino dell Unione Matematica Italiana. Sezione B, vol. 8, no. 2, pp. 289-303, 1994.

[5] S.-T. Wu and L.-Y. Tsai, "On global existence and blow-up of solutions for an integro-differential equation with strong damping," Taiwanese Journal of Mathematics, vol. 10, no. 4, pp. 9791014, 2006.

[6] S.-T. Wu, "Exponential energy decay of solutions for an integrodifferential equation with strong damping," Journal of Mathematical Analysis and Applications, vol. 364, no. 2, pp. 609-617, 2010.

[7] V. Georgiev and G. Todorova, "Existence of a solution of the wave equation with nonlinear damping and source terms," Journal of Differential Equations, vol. 109, no. 2, pp. 295-308, 1994.

[8] H. A. Levine, "Instability and nonexistence of global solutions to nonlinear wave equations of the form Dutt=Au+F(u)," Transactions of the American Mathematical Society, vol. 192, pp. 1-21, 1974.

[9] H. A. Levine, "Some additional remarks on the nonexistence of global solutions to nonlinear wave equations," SIAM Journal on Mathematical Analysis, vol. 5, no. 1, pp. 138-146, 1974.

[10] H. A. Levine, S. R. Park, and J. Serrin, "Global existence and global nonexistence of solutions of the Cauchy problem for a nonlinearly damped wave equation," Journal of Mathematical Analysis and Applications, vol. 228, no. 1, pp. 181-205, 1998.

[11] E. Vitillaro, "Global nonexistence theorems for a class of evolution equations with dissipation," Archive for Rational Mechanics and Analysis, vol. 149, no. 2, pp. 155-182, 1999.

[12] G. Kirchhoff, Vorlesungen Über Mechanik, Teubner, Leipzig, Germany, 1883.

[13] K. Ono, "Blowing up and global existence of solutions for some degenerate nonlinear wave equations with some dissipation," vol. 30, no. 7, pp. 4449-4457.

[14] K. Ono, "Global existence, decay, and blowup of solutions for some mildly degenerate nonlinear Kirchhoff strings," Journal of Differential Equations, vol. 137, no. 2, pp. 273-301, 1997.

[15] S. T. Wu and L. Tsai, On global solutions and blow-up solutions for a nonlinear viscoelastic wave equation with nonlinear damping, National Chengchi University, 2004.

[16] S. T. Wu and L. Y. Tsai, "Blow-up of solutions for some nonlinear wave equations of Kirchhoff type with some dissipation," Nonlinear Analysis. Theory, Methods \& Applications, vol. 65, no. 2, pp. 243-264, 2006. 
[17] S.-T. Wu and L.-Y. Tsai, "On a system of nonlinear wave equations of Kirchhoff type with a strong dissipation," Tamkang Journal of Mathematics, vol. 38, no. 1, pp. 1-20, 2007.

[18] R. $\mathrm{Xu}$ and Y. Yang, "Finite time blow-up for the nonlinear fourth-order dispersive-dissipative wave equation at high energy level," International Journal of Mathematics, vol. 23, no. 5, Article ID 12500607, 2012.

[19] M. Reed, Abstract non-linear wave equations, Lecture Notes in Mathematics, Vol. 507, Springer-Verlag, Berlin-New York, 1976.

[20] K. Agre and M. A. Rammaha, "Systems of nonlinear wave equations with damping and source terms," Differential and Integral Equations. An International Journal for Theory \& Applications, vol. 19, no. 11, pp. 1235-1270, 2006.

[21] C. O. Alves, M. M. Cavalcanti, V. N. Domingos Cavalcanti, M. A. Rammaha, and D. Toundykov, "On existence, uniform decay rates and blow up for solutions of systems of nonlinear wave equations with damping and source terms," Discrete and Continuous Dynamical Systems - Series S, vol. 2, no. 3, pp. 583-608, 2009.

[22] B. Said-Houari, "Global nonexistence of positive initial-energy solutions of a system of nonlinear wave equations with damping and source terms," Differential and Integral Equations, vol. 23, no. 1-2, pp. 79-92, 2010.

[23] M. A. Rammaha and S. Sakuntasathien, "Global existence and blow up of solutions to systems of nonlinear wave equations with degenerate damping and source terms," Nonlinear Analysis. Theory, Methods \& Applications, vol. 72, no. 5, pp. 26582683, 2010.

[24] G. Li, Y. Sun, and W. Liu, "Global existence, uniform decay and blow-up of solutions for a system of Petrovsky equations," Nonlinear Analysis. Theory, Methods \& Applications, vol. 74, no. 4, pp. 1523-1538, 2011.

[25] M. A. Rammaha and S. Sakuntasathien, "Critically and degenerately damped systems of nonlinear wave equations with source terms," Applicable Analysis: An International Journal, vol. 89, no. 8, pp. 1201-1227, 2010.

[26] X. Han and M. Wang, "Global existence and blow-up of solutions for a system of nonlinear viscoelastic wave equations with damping and source," Nonlinear Analysis. Theory, Methods of Applications, vol. 71, no. 11, pp. 5427-5450, 2009.

[27] J. Wu, S. Li, and S. Chai, "Existence and nonexistence of a global solution for coupled nonlinear wave equations with damping and source," Nonlinear Analysis. Theory, Methods \& Applications, vol. 72, no. 11, pp. 3969-3975, 2010.

[28] B. Said-Houari, S. A. Messaoudi, and A. Guesmia, "General decay of solutions of a nonlinear system of viscoelastic wave equations," Nonlinear Differential Equations and Applications NoDEA, vol. 18, no. 6, pp. 659-684, 2011.

[29] S. A. Messaoudi and B. Said-Houari, "Global nonexistence of positive initial-energy solutions of a system of nonlinear viscoelastic wave equations with damping and source terms," Journal of Mathematical Analysis and Applications, vol. 365, no. 1, pp. 277-287, 2010.

[30] B. Said-Houari, "Exponential growth of positive initial-energy solutions of a system of nonlinear viscoelastic wave equations with damping and source terms," Zeitschrift für Angewandte Mathematik und Physik, vol. 62, no. 1, pp. 115-133, 2011.

[31] W. Liu, "Global existence and uniform decay of solutions for a system of wave equations with dispersive and dissipative terms," Frontiers of Mathematics in China, vol. 5, no. 3, pp. 555-574, 2010.
[32] W. Liu, "Global existence, asymptotic behavior and blow-up of solutions for coupled Klein-Gordon equations with damping terms," Nonlinear Analysis. Theory, Methods \& Applications, vol. 73, no. 1, pp. 244-255, 2010.

[33] F. Liang and H. Gao, "Exponential energy decay and blow-up of solutions for a system of nonlinear viscoelastic wave equations with strong damping," Boundary Value Problems, 2011:22, 19 pages, 2011.

[34] S. A. Messaoudi and N.-e. Tatar, "Uniform stabilization of solutions of a nonlinear system of viscoelastic equations," Applicable Analysis: An International Journal, vol. 87, no. 3, pp. 247-263, 2008.

[35] M. de Lima Santos, "Decay rates for solutions of a system of wave equations with memory," Electronic Journal of Differential Equations, No. 38, 17 pages, 2002.

[36] L. Liu and M. Wang, "Global existence and blow-up of solutions for some hyperbolic systems with damping and source terms," Nonlinear Analysis. Theory, Methods \& Applications, vol. 64, no. 1, pp. 69-91, 2006.

[37] B. Said-Houari, "Global existence and decay of solutions of a nonlinear system of wave equations," Applicable Analysis: An International Journal, vol. 91, no. 3, pp. 475-489, 2012.

[38] S.-T. Wu, "On decay and blow-up of solutions for a system of nonlinear wave equations," Journal of Mathematical Analysis and Applications, vol. 394, no. 1, pp. 360-377, 2012.

[39] M. Nakao, "A difference inequality and its application to nonlinear evolution equations," Journal of the Mathematical Society of Japan, vol. 30, no. 4, pp. 747-762, 1978.

[40] Y. Wang, "A global nonexistence theorem for viscoelastic equations with arbitrary positive initial energy," Applied Mathematics Letters, vol. 22, no. 9, pp. 1394-1400, 2009.

[41] J. Ma, C. Mu, and R. Zeng, "A blow up result for viscoelastic equations with arbitrary positive initial energy," Boundary Value Problems, 2011:6, 10 pages, 2011.

[42] R. Zeng, C. Mu, and S. Zhou, "A blow-up result for Kirchhofftype equations with high energy," Mathematical Methods in the Applied Sciences, vol. 34, no. 4, pp. 479-486, 2011.

[43] R. Xu, Y. Yang, and Y. Liu, "Global well-posedness for strongly damped viscoelastic wave equation," Applicable Analysis: An International Journal, vol. 92, no. 1, pp. 138-157, 2013.

[44] R. Xu, Y. Yang, S. Chen, J. Su, J. Shen, and S. Huang, "Nonlinear wave equations and reaction-diffusion equations with several nonlinear source terms of different signs at high energy level," The ANZIAM Journal. The Australian \& New Zealand Industrial and Applied Mathematics Journal, vol. 54, no. 3, pp. 153-170, 2013.

[45] G. Filippo and S. Marco, "Global solutions and finite time blow up for damped similinear wave equations," Ann. I. H. PoincaréAn, vol. 7, pp. 675-702, 2006.

[46] S. A. Messaoudi, "Blow-up of positive-initial-energy solutions of a nonlinear viscoelastic hyperbolic equation," Journal of Mathematical Analysis and Applications, vol. 320, no. 2, pp. 902915, 2006. 


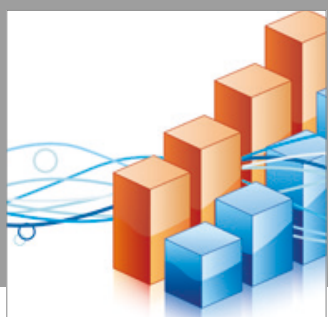

Advances in

Operations Research

vatersals

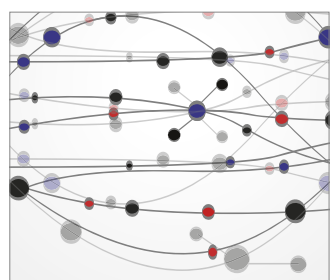

\section{The Scientific} World Journal
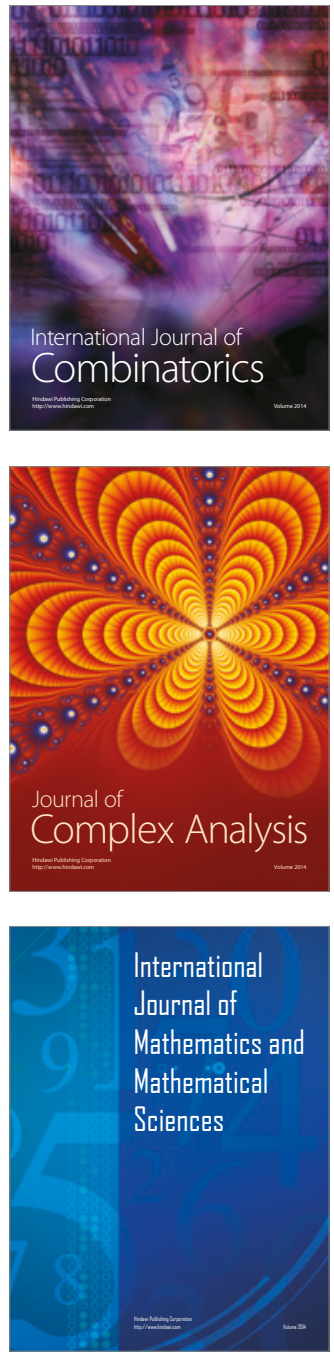
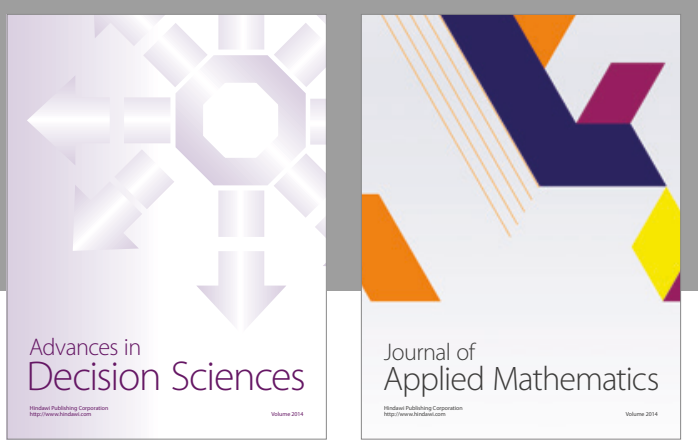

Algebra

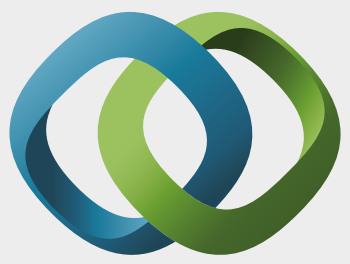

\section{Hindawi}

Submit your manuscripts at

https://www.hindawi.com
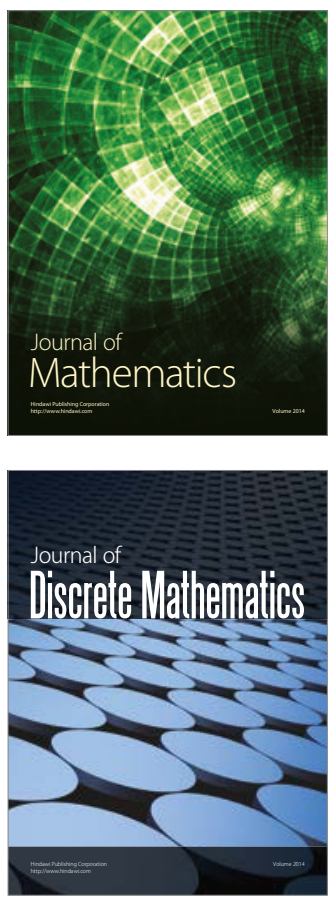

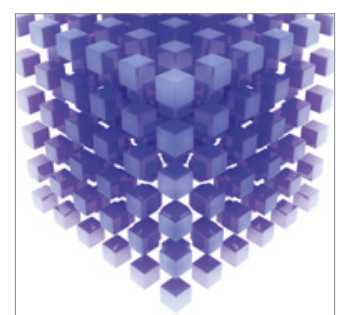

Mathematical Problems in Engineering
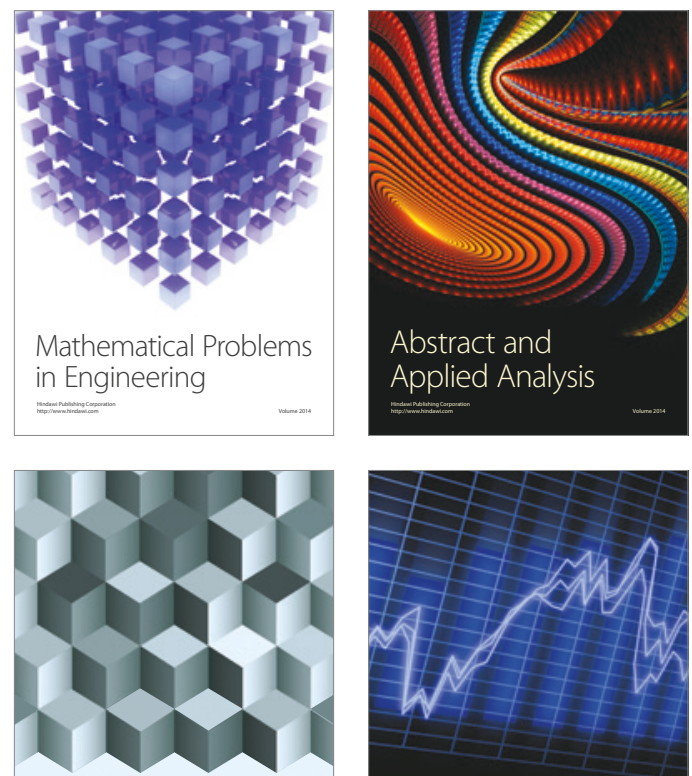

Journal of

Function Spaces

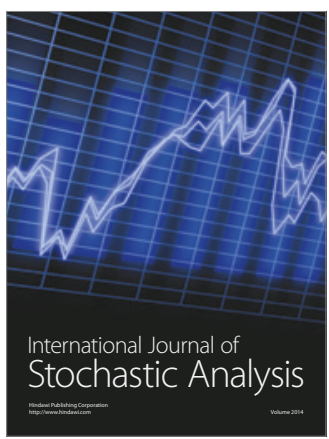

Probability and Statistics
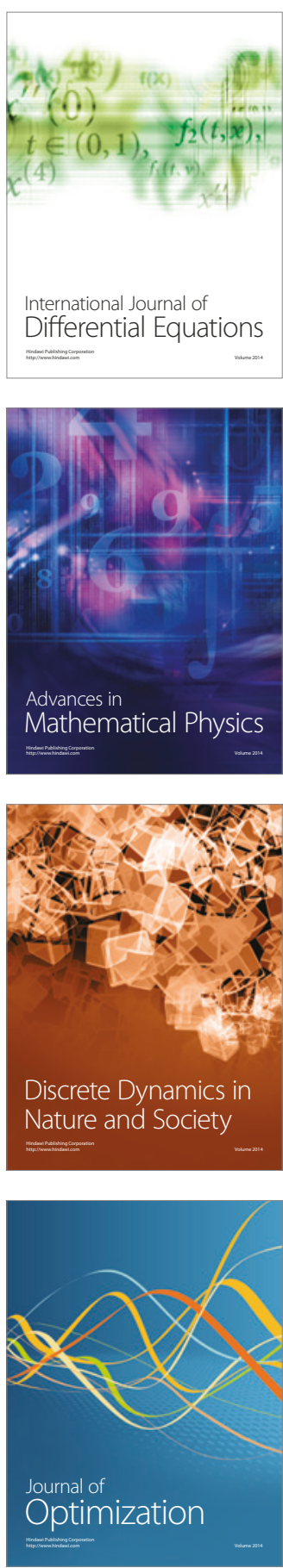